\title{
Seasonal variations of subsurface seismic velocities monitored by the SEIS-InSight seismometer on Mars
}

\author{
N. Compaire ${ }^{1}$, L. Margerin ${ }^{2}$, M. Monnereau ${ }^{2}$, R.F. Garcia ${ }^{1,2}$,
}

L. Lange ${ }^{3}$, M. Calvet ${ }^{2}$, N. L. Dahmen ${ }^{4}$, S. C. Sthler ${ }^{4}$, N. Mueller ${ }^{5}$, M. Grott ${ }^{5}$, P. Lognonné ${ }^{6}$, T. Spohn ${ }^{5}$, W. B. Banerdt ${ }^{7}$

${ }^{1}$ Institut Suprieur de l'Aronautique et de l'Espace SUPAERO, 10 Avenue Edouard Belin, 31400 Toulouse, France.

${ }^{2}$ Institut de Recherche en Astrophysique et Plantologie, Universit Toulouse III Paul Sabatier,

CNRS, CNES, 14 Av. E. Belin, 31400, Toulouse, France.

${ }^{3}$ Laboratoire de Mtorologie Dynamique/Institut Pierre Simon Laplace (LMD/IPSL), Sorbonne Universit,

CNRS, cole Polytechnique, cole Normale Suprieure (ENS), Campus Pierre et Marie Curie BC99, Paris, France.

${ }^{4}$ Institute of Geophysics, ETH Zurich, Zurich, Switzerland.

${ }^{5}$ German Aerospace Center (DLR), Institute of Planetary Research, Rutherfordstr. 2, 12489 Berlin, Germany.

${ }^{6}$ Universit de Paris, Institut de physique du globe de Paris, CNRS, F-75005 Paris, France.

${ }^{7}$ Jet Propulsion Laboratory, California Institute of Technology, Pasadena, CA 91109, USA.

\section{SUMMARY}

The SEIS seismometer deployed at the surface of Mars in the framework of the NASAInSight mission has been continuously recording the ground motion at Elysium Planitia for more than one martian year. In this work, we investigate the seasonal variation of the near surface properties using both background vibrations and a particular class of highfrequency seismic events. We present measurements of relative velocity changes over one martian year and show that they can be modeled by a thermoelastic response of the Martian regolith. Several families of high-frequency seismic multiplets have been observed at

various periods of the martian year. These events exhibit complex, repeatable waveforms with an emergent character and a coda that is likely composed of scattered waves. Tak- 
ing advantage of these properties, we use coda wave interferometry to measure relative travel-time changes as a function of the date of occurrence of the quakes. While in some families a stretching of the coda waveform is clearly observed, in other families we observe either no variation or a clear contraction of the waveform. These various behaviors correspond to different conditions of illumination at the InSight landing site, depending on the season. Measurements of velocity changes from the analysis of background vibrations above $5 \mathrm{~Hz}$ are consistent with the results from coda wave interferometry. We identify a frequency band structure in the power spectral density that can be tracked over hundreds of days. This band structure is the equivalent in the frequency domain of an autocorrelogram and can be efficiently used to measure relative travel-time changes as a function of frequency. We explain how the PSD analysis allows us to circumvent the contamination of the measurements by the Lander mode excitation which is ineyitable in the time domain. The observed velocity changes can be adequately modeled by the thermoelastic response of the regolith to the time-dependent incident solar flux at the seasonal scale. In particular, the model captures the time delay between the surface temperature variations and the velocity changes in the subsurface. Our observations could serve as a basis for a joint inversion of the seismic and thermal properties in the first 20 meters below InSight.

Key words: Seismic interferometry - Seismic noise - Coda waves - Planetary interiors.

\section{INTRODUCTION}

The SEIS (Seismic Experiment for Interior Structure) seismometer (Lognonn et al. 2019) deployed on the surface of Mars in the framework of the NASA's InSight (Interior Exploration using Seismic Investigations, Geodesy and Heat Transport) mission provides an unprecedented opportunity to study the properties of the Martian regolith and the nature of the seismic wavefield at Elysium Planitia (Banerdt et al. 2020; Lognonn et al. 2020).

Monitoring the properties of the subsurface with seismic waves is a lively field of research. Taking advantage of the fact that repetitive seismic sources produce nearly identical waveforms, Poupinet et al. (1984) introduced the doublet method to quantify temporal changes in seismic velocities in the crust. These authors were the first to observe linear trends in the time-delays measured as a function 
of the lapse-time in the coda, when comparing waveforms of small events that occurred respectively before and after a large earthquake in California. They interpreted this observation as the result of a velocity change in the crust caused by strong ground motions. They proposed that a uniform perturbation of background velocity $\delta v / v$ would not modify the path of multiply-scattered coda waves but would affect their phase delay in a way such that it increases $(\delta v / v<0)$ or decreases $(\delta v / v>0)$ linearly with the lapse-time in the coda, as compared to the unperturbed case. Since Poupinet et al. (1984)'s breakthrough, the doublet method aka coda wave interferometry (CWI) has been substantiated by multiple-scattering theories and generalized to more complex, local changes in propagation properties (Snieder et al. 2002; Pacheco \& Snieder 2005; Larose et al. 2010; Planès et al. 2014; SensSchönfelder \& Eulenfeld 2019). It was shown in particular that a slight shift in the source position does not result in a phase delay in the coda but rather in a loss of correlation of the waveforms. In fact, it is well established that the band-passed signals emitted by two sources distant by more than half the central wavelength are almost entirely uncorrelated in a multiple-scattering medium (Akkermans \& Montambaux 2007). Sens-Schönfelder \& Wegler (2006) showed that the principle of CWI could also be fruitfully applied to monitoring using background vibrations. The basic idea is to use the empirical Green's functions (GF) retrieved by noise cross-correlations (Shapiro 2005) at different dates as a sequence of seismic doublets (also called multiplets). In the case of a diffuse ambient noise field (Lobkis \& Weaver 2001; Weaver \& Lobkis 2004), the empirical GF contains all direct and reflected body and surface waves, as well as coda waves, to which CWI can be, in turn, applied. Following the work of Sens-Schönfelder \& Wegler (2006), monitoring techniques based on ambient noise correlations have been collectively referred to as Passive Image Interferometry (PII).

Sens-Schnfelder \& Larose (2008) successfully applied PII to seismic records of the Apollo 17 Lunar Seismic Profiling Experiment and detected velocity variations in the Lunar regolith induced by periodic changes in the inflow of solar energy. Their work presents a convincing demonstration of the applicability of seismic interferometry techniques in extraterrestrial conditions and a significant illustration of the potential of the methods for planetary seismology (see also Tanimoto et al. (2008)). As noted by Sens-Schnfelder \& Larose (2008), the conditions on the Moon were well adapted to the application of seismic interferometry. The lunar subsurface exhibits much more intense scattering and far less attenuation than Earth (Dainty \& Toksz 1981), facilitating the fast convergence of ambient noise cross-correlations towards the Green's function of the medium (Larose et al. 2008). Moreover, the origin of the background vibration of the Lunar soil is well known and appears to be mainly related to the numerous thermal events, which provide an excellent source of diffuse seismic illumination (Larose et al. 2005).

On Mars, the origin and nature of the background vibrations are still under investigation. Scat- 
tering, while not as intense as on the Moon, largely dominates attenuation on Mars (Lognonn et al. 2020; van Driel et al. 2021; Menina et al. 2021). Several studies have applied seismic interferometry techniques to the record of the SEIS-InSight instrument for imaging applications (Suemoto et al. 2020; Deng \& Levander 2020; Compaire et al. 2021; Schimmel et al. 2021; Knapmeyer-Endrun et al. 2021), supporting the idea that auto-correlations functions of background vibrations above $1 \mathrm{~Hz}$ are composed of waves propagating in the Martian crust. In the present work, we investigate one Martian year (equal to 668.6 Martian days, called Sol and corresponding to 24h40) of seismic data and report observations of seasonal-thermally induced seismic velocity changes from both high-frequency seismic multiplets and background vibrations in the 5-10 Hz frequency band. In section 2, we present an application of CWI to 8 families of diffuse seismic multiplets waveforms and show a gradual transition in the relative traveltimes changes measured across the InSight mission time-line, reflecting a decrease of the seismic velocity at the beginning of the period of study and an increase of the velocity at the end. In section 3 we present a frequency-based version of PII that enables the measurement of temporal variations in the background signal of SEIS, in spite of the strong disturbances generated by the Insight lander which completely corrupt time-domain correlograms. The method can be briefly summarized as follows. We first identify characteristic oscillations in the power-spectral density (PSD) of ambient vibrations, that can be tracked over the entire duration of the mission. Because of their visual appearance in a frequency/time diagram, these oscillations will be suggestively referred to as "band structure" and can be clearly observed between 5 and $10 \mathrm{~Hz}$ on the horizontal components of SEIS. Next, we show that the band structure presents seasonal variations that can be interpreted as velocity changes, consistent with those obseryed in the seismic events. In section 4 we show that both the amplitude and the delay of the observed velocity changes with the temperature signal can be modeled by a thermoelastic response of the first few meters of the Martian regolith. In section 5 we examine critically the results of our model and suggest possible improvements and future works.

\section{OBSERVATIONS FROM SUPER-HIGH-FREQUENCY EVENTS (SF)}

\subsection{The families of seismic multiplets}

In this section, we focus on a particular class of high-frequency seismic events recorded by the SEISInSight seismometer, which have been identified and described by Dahmen et al. (2021a). Based on their characteristics the MarsQuake Service (MQS) (InSight Marsquake Service 2021) assigned them the name "Super-High-Frequency" events, abbreviated as "SF". These events have a typical duration of approximately 20s with a peak of energy between $5 \mathrm{~Hz}$ and $10 \mathrm{~Hz}$ mainly on the horizontal components. Their waveforms being strongly diffuse, no seismic phases can be identified. Therefore, 
in the MQS nomenclature the SF events can only be assigned a quality $\mathrm{C}$ or D depending on their signal-to-noise ratio. With more than 780 detections the SF family is the largest class of events of the InSight mission. Dahmen et al. (2021a) report the high similarity between the waveforms of several SF events. With a template matching technique the authors have identified 16 distinct clusters of SF events with a high correlation coefficient. In this study we performed a clustering of 793 SF events (170 quality C and 623 quality D) with a methodology similar to Dahmen et al. (2021a). We present in this section the result of this classification.

Because they offer an exhaustive temporal coverage, we use the 20 sample-per-second (sps) records of the Very-BroadBand (VBB) sensor of SEIS (InSight Mars SEIS Data Service 2019). To construct our database of 3-components SF waveforms we use the start and end time of the 50 secondstime window containing the event, as reported in the MQS catalogue (InSight Marsquake Service 2021). Using the pre-processing described in Compaire et al. (2021) we remove from the raw data the tick noise (cross-talk between temperature acquisition and seismic channels) and the glitches. After the removal of the instrumental response and the rotation onto the local geographical coordinate system (Z : upward vertical, N : South-North and E : West-East) we filter the SF waveforms between 7 $\mathrm{Hz}$ and $9 \mathrm{~Hz}$, as suggested by Dahmen et al. (2021a).

We analyze separately the quality $\mathrm{C}$ and $\mathrm{D}$ SF events and build a correlation matrix $C m$ for each category as follows. For each event pair $(i, j)$, the matrix element $C m_{i j}$ is defined as the maximum of the full cross-correlation function of the waveforms. From the upper triangular coefficients of $C_{m}$, we identify events with correlation higher than a pre-defined threshold $T$. We choose $T=0.6$ and $T=0.5$ for quality $\mathrm{C}$ and $\mathrm{D}$ events, respectively. All events are chronologically inspected one after the other. For each event $i$, we obtain a list $L_{i}$ of $N_{i}$ events with a similar waveform. If the list $L_{i}$ is empty, event $i$ forms a singleton. If the list $L_{i}$ is non-empty, then $i$ and all events in $L_{i}$ are collected in a common family $F_{i}$. If the event $k$ is in $L_{i}$, then all the events in $L_{k}$ not present in $L_{i}$ are added to the family $F_{i}$. When an event has been assigned to a family (even a singleton) it cannot re-appear in any other family. It is therefore deleted from all the lists that have not yet been explored. At the end of this process we only retain families with more than $5 \%$ of the total number of events for each quality.

The results of this classification are presented in Figure 1.A. For the SF events of quality C and D, respectively three and five families were identified. We label each family based on its quality (C or D) and its order of appearance during the InSight mission time-line ( $\mathrm{C} 0, \mathrm{C} 1$ and $\mathrm{C} 2$ for quality $\mathrm{C}$ events and D0, D1, D2, D3 and D4 for quality D events). Figure 1.A shows a scatter plot of the temporal distribution of events composing each family in the Local Mean Solar Time (LMST)-Sol (martian day) plane. Our classification is very consistent with the one proposed by Dahmen et al. (2021a). Our families C0, C1 and C2 correspond respectively to families n 1, 4 and 16 of Dahmen et al. (2021a). Our 
families D0, D1, D2, D3 and D4 correspond respectively to families n 3, 5, 8, 12 and 14 of Dahmen et al. (2021a).

We give in Appendix A the list of the events present in each family. In Appendix A1 we perform a Signal-to-Noise Ratio (SNR) analysis on all these events sorted by family. On quality D events the SNR is maximal between 7 and $9 \mathrm{~Hz}$. For all events the SNR is higher on the East component. We therefore conducted the rest of this analysis with the same configuration for all events, i.e. the East component filtered between 7 and 9Hz.

Figure 1.B shows the waveforms of the SF events in family C0 ordered by increasing Sols. The waveforms for all the others families are given in Appendix A2. The waveforms are aligned on the first event of the family. We can see that the events of each family have indeed extremely similar waveforms. According to Dahmen et al. (2021a), this suggests that these events are produced by the same sources repeating for several weeks. We can therefore speak here of seismic multiplets. The fact that these events occur systematically when the atmospheric temperature gradients are the highest points to thermal cracking occurring in the local regolith. The possibility that these cracks come from the lander is not totally excluded by Dahmen et al. (2021a). However, unlike the well-identified lander cracks, called "donks" (Ceylan et al. 2021; Compaire et al. 2021), the SF events do not excite any of the lander modes, thus favoring a seismic source outside of the lander system.

We remark that the events of family $\mathrm{C} 0$ in figure $1 . \mathrm{B}$ show a clear stretching of their waveforms with increasing Sols. In section 2.2 we use the doublet method of Poupinet et al. (1984) to measure in each SF family the relative traveltime changes at the origin of this waveform stretching.

\subsection{Measure of relative traveltime changes}

Several techniques have been developed to measure traveltime changes in the coda waveforms of either seismic multiplets or cross-correlations of background vibrations computed at different dates (Liu et al. 2010). In this study we adopt the Moving-Window Cross-Spectral technique (MWCS) introduced by Poupinet et al. (1984) for seismic doublets, following the process described by Clarke et al. (2011). In this séction we briefly outline the method. We refer the reader to Clarke et al. (2011) (and references therein) for more details.

Let us consider a seismic doublet. In the case of a homogeneous velocity variation in the medium occurring between the two events, the effect on the arrival time of the waves is proportional to the time spent in the medium. If we compare the codas of the two events, the time-delay induced in the waveforms will increase linearly with the time in the coda (Poupinet et al. 1984, 2008). The principle of the MWCS method is to calculate the time-delay between two diffuse waveforms in sliding windows and then to calculate the linear regression of the time-delays as a function of the lapse-time. The time- 


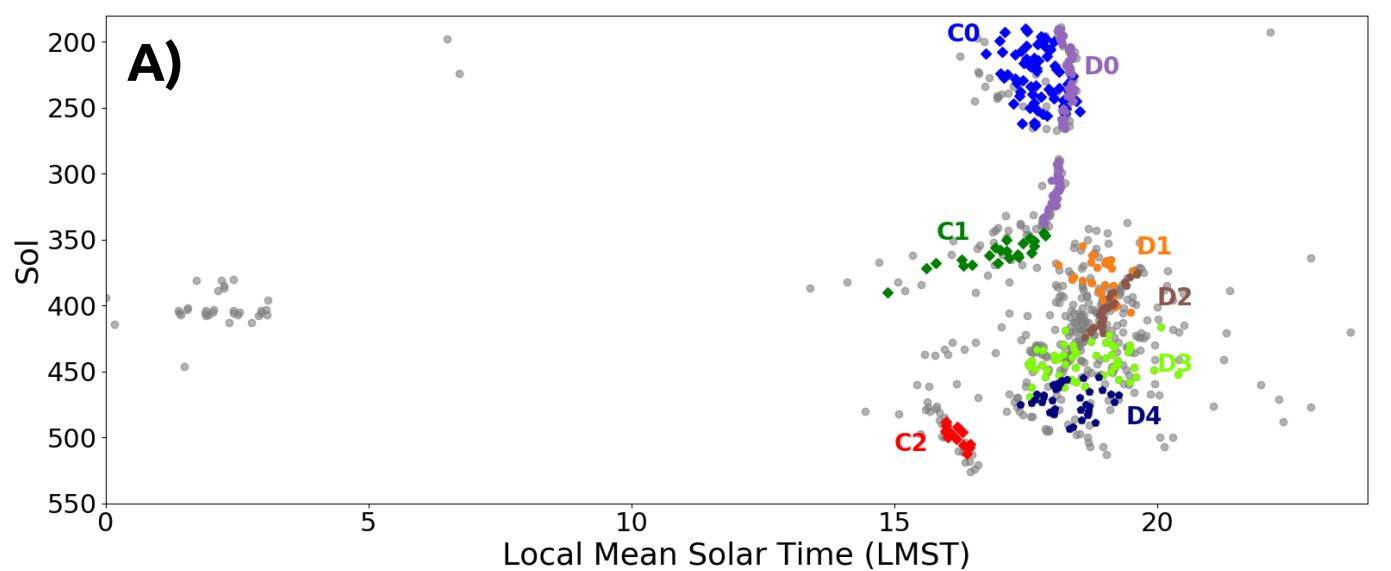

\section{B)}

Co

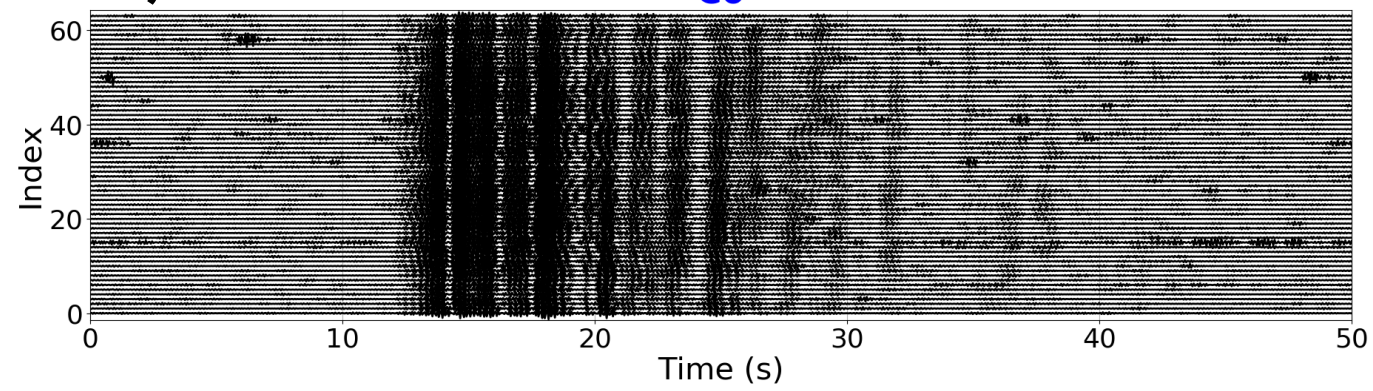

Figure 1. A) Temporal distribution of the Super High-Frequency events (SF) used in this study. The horizontal and vertical axes show the hour (LMST, see text) and day (Sol, see text) of occurrence of the event. Each family of SF events is identified with a particular color and labeled with a code (see text). Events that define their own family are shown in gray. B) Waveforms of the East component filtered between $7 \mathrm{~Hz}$ and $9 \mathrm{~Hz}$ of the SF events in the family $\mathrm{C} 0$ ordered by increasing Sols. Notice the stretching of the waveforms with increasing time of occurrence.

delay $d t^{i}$ in the sub-window $i$ is computed in the spectral domain using the linear relationship between the phase of the cross-spectrum and the time-delay : $\phi^{i}(f)=2 \pi \times d t^{i} \times f$ with $f$, the frequency in $\mathrm{Hz}$ and $\phi^{i}(f)$, the phase of the cross-spectrum $X^{i}(f)=F_{1}^{i}(f) F_{2}^{i}(f)^{*}=\left|X^{i}(f)\right| e^{i \phi^{i}(f)} \cdot F_{1}^{i}$ and $F_{2}^{i}$ designate the Fourier transforms of the events waveforms in window $i$ and the symbol * denotes complex conjugation. The time-delay $d t^{i}$ is subsequently estimated from a weighted linear regression of the cross-phase $\phi^{i}(f)$ in a given frequency band. The weights are functions of the coherence $C^{i}(f)$ between the two waveforms in the sub-window $i$, with the coherence defined as :

$$
C^{i}(f)=\frac{\left|\overline{X^{i}(f)}\right|}{\sqrt{\overline{\left|F_{1}^{i}(f)\right|^{2} \cdot\left|F_{2}^{i}(f)\right|^{2}}}}
$$

With the overlines designating a smoothing. Here we use sliding hanning windows of 3 samples (corresponding to a half-width of $0.5 \mathrm{~Hz}$ ). 
Once all individual time delays have been estimated, the global relative traveltime change $d t / t$ between a reference and a current waveform is simply deduced from a linear regression of the $d t^{i} \mathbf{s}$ as a function of the lapse-time in the coda. This procedure is illustrated in Figure 2.A.B.C for the $\mathrm{SF}$ event T0203a in family $\mathrm{C} 0$. The reference waveform is defined as the average waveform after alignment of the time-series by cross-correlation. For the evaluation of the $d t^{i}$, we choose a sliding window duration of 2 seconds with an overlap of $50 \%$. The time interval where the seismic energy significantly exceeds the noise level starts approximately at 13 seconds and ends approximately at 25 seconds. We will refer to this interval as the event window. For this example, we perform a linear regression of the cross-phase in the 7-9 Hz band. In Figure 2.A we show the coherence between the reference waveform and the waveform of the T0203a event in each sub-window. As defined in equation 1 the coherence is function of the frequency. Therefore, we represent in Figure 2.A the average coherence in the frequency band of study. We see that during the event window the coherence is maximum $(>0.9)$ and that the measured time-delays (Figure 2.C) show a clear linear trend. In Figure 2.C the slope of the relative traveltime change $d t / t$ as a function of lapse-time is negative in the event window, thereby indicating a compression of the current waveform compared to the reference. This implies that event T0203a has propagated in a medium with a velocity slightly higher than the average velocity in the time period of the family $C_{0}$.

The relative traveltime changes $d t / t$ obtained with the procedure previously described are shown in red in Figure 2.D for all the events of the family C0, as a function of the Sol. In monitoring studies, it is a common practice to represent $-d t / t$ rather than $d t / t$ because in the case of a homogeneous seismic velocity perturbation in the medium we have: $d v / v=-d t / t$ (where $d v / v$ is the relative seismic velocity change in the medium). These measurements show a clear linear drift in traveltime of about $0.8 \%$ between Sol 190 and Sol 263 . Furthermore, $d t / t$ changes sign approximately in the middle of the time window of $\mathrm{C} 0$ detections. Keeping in mind that the measurements are performed with respect to the average $\mathrm{C} 0$ waveform, these observations suggest that the daily velocity change is close to uniform during the time period extending from Sol 193 to Sol 263.

In order to test the sensitivity of the global traveltime changes to the frequency band, we also applied the measurement procedure to the waveforms of the family $\mathrm{C} 0$ filtered in the 5-7 $\mathrm{Hz}$ band. The derived $d t / t$ are shown in black in Figure 2.D. The linear trend is also clearly observed in the 5-7 $\mathrm{Hz}$ band but the slope appears to be lower in absolute value. This observation will be put into perspective when compared with the measurements made in the background vibrations in section 3.2.

The results of the MWCS analysis for all SF events of all families are shown in Figure 3 for the 7-9 $\mathrm{Hz}$ band. In Figure 3.A, the panels corresponding to each family are ordered from left to right and from top to bottom by time of appearance in the course of the InSight mission. The main outcome 

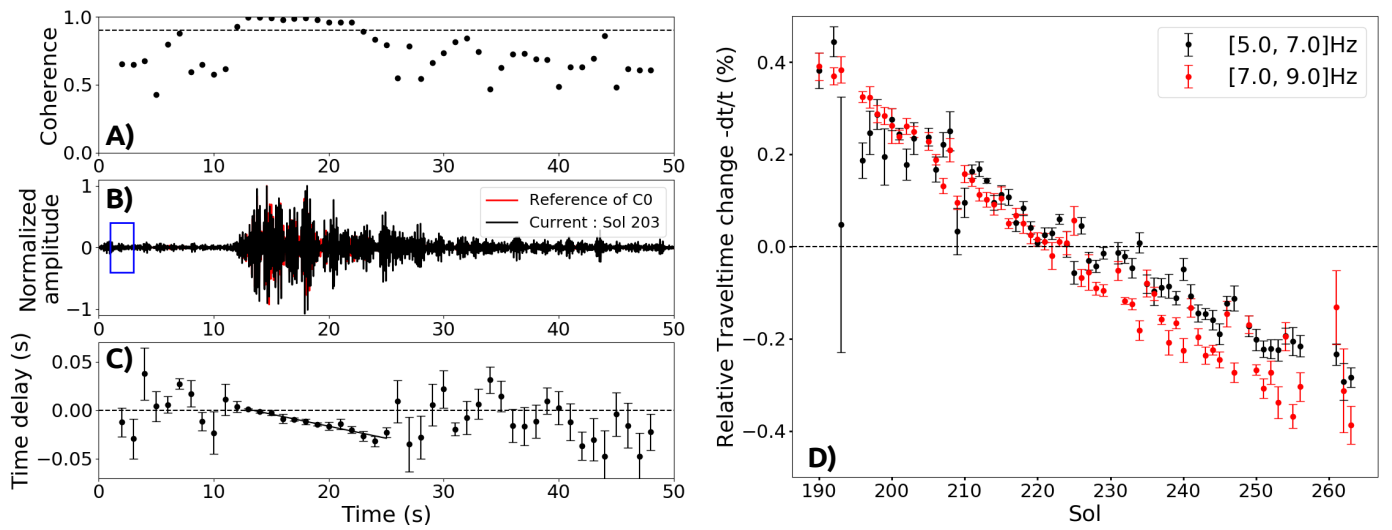

Figure 2. Illustration of the Moving-Window Cross-Spectrum analysis on the family $\mathrm{C} 0$. A) Coherence between the average waveform of the family C0 (reference) and the event T0203a (current) in 2 seconds-sub-windows with a 1 second overlap. The dotted horizontal line indicates a coherence of 0.9. B) Red: Reference waveform for the family C0 (red curve). Black: waveform of the SF event T0203b. A band-pass filter has been applied between $7 \mathrm{~Hz}$ and $9 \mathrm{~Hz}$ and the amplitudes have been normalized by the maximum. The blue rectangle delimits the first sub-window. C) Measured time-delays between the reference and current waveforms following the method of Clarke et al. (2011). The slope of the linear regression of the time-delays during the event window (13-25 seconds) provides the relative traveltime change $\mathrm{dt} / \mathrm{t}$ between the reference and current waveforms. D) Relative traveltime change $-d t / t$ measured across the family $\mathrm{C} 0$ in the 7-9 $\mathrm{Hz}$ band (red points) and the $5-7 \mathrm{~Hz}$ band (black points).

of the analysis of the SF events is the observation of a gradual transition in the measurements of $-d t / t$ from a strong stretching of the SF waveforms in the first families $(C 0, \mathrm{D} 0$ - from Sol 190 to Sol 350) to a strong compression of the SF waveforms in the last families (D3, D4, C2 - from Sol 420 to Sol 512). In order to better visualize the transition, we have attempted the reconstruction of a globally continuous function relating the relative traveltime change to the Sol. The result is shown in Figure 3.B where we have reported the measurements from Figure 3.A on the same axis (points without error bars). We can see that over the period running from Sol 190 to Sol 266 the families C0 and D0 show slightly different trends. This can be explained by the difference in SNR between the two families ( $S N R \sim 4$ for family C0 and $S N R \sim 1.5$ for family D0 between $7 \mathrm{~Hz}$ and $9 \mathrm{~Hz}$ ). Indeed we know that the accuracy of the MWCS analysis depends greatly on the SNR (Clarke et al. 2011). However, the difference in $d t / t$ between the two families remains small because the measured variations are large. To bring the measurements obtained from the various families into continuity, we added a family-dependent corrective constant (indicated in Figure 3.B) to the $d t / t$ (points with error bars). In doing so, we gave priority to events of quality $\mathrm{C}$ and imposed that $d t / t=0 \%$ for the first event of family $\mathrm{C} 0$. 
The main information we infer from Figure 3.B is 1) the peak-to-peak amplitude of the relative traveltime variation which is about $2 \%, 2$ ) the time of occurrence of the minimum $d t / t$ which approximately lies between Sol 300 and Sol 400.

Between Sol 500 and Sol 800, a change in atmospheric conditions caused the noise level recorded at night to increase considerably (see Figure 5). During this period no SF events could be detected. In order to obtain a curve of velocity variations over the whole martian year (668.6 Sols) we also analyzed the background vibrations recorded by SEIS at 20 sample-per-second (sps) from Sol 180 to Sol 870.

\section{OBSERVATIONS FROM BACKGROUND VIBRATION}

\subsection{The frequency band structure}

The technique allowing the monitoring of velocity changes using continuous records of background vibration is called Passive Image Interferometry (PII) (Sens-Schönfelder \& Wegler 2006). This technique is based on the comparison of Greens functions retrieved at different times by cross-correlation of the background vibration recorded at two distinct stations. PII has also been applied in single-station configuration to monitor volcanic activity or velocity variations induced by stress redistribution after an earthquake (De Plaen et al. 2016; Hobiger et al. 2014). In that case the cross-correlogram between two seismic sensors is replaced by an auto-correlogram.

For monitoring applications, the key issue is not the perfect reconstruction of the Green's functions but rather the stability of the background sources in terms of spatial distribution and frequency content (Hadziioannou et al. 2009; Zhan et a1. 2013). For this reason, the record from the InSight-SEIS seismometer is a complicated case of study. Indeed, the background signal is not well characterized, partially non-seismic (Stutzmann et al. 2021; Ceylan et al. 2021) and contains a lot of continuous and transients perturbations eoming from the local environment (Scholz et al. 2020; Panning et al. 2020; Lognonn et al. 2020). In particular, all studies focusing on temporal variations have to deal with the presence of numerous lander modes visible in the spectra of background vibrations above $1 \mathrm{~Hz}$. These lander modes show strong diurnal and seasonal variations correlated with the changes in local atmospheric conditions (Dahmen et al. 2021b). The challenge is therefore to discriminate the potential temporal variations of the subsurface elastic parameters from the temporal variations of the lander modes.

Because lander modes are readily identified in the frequency domain, it is a good strategy to work in frequency to discriminate between lander-related signals and others type of signals. By the Wiener-Khintchine theorem, we know that the Auto-Correlation Function (ACF) of a stationary ran- 

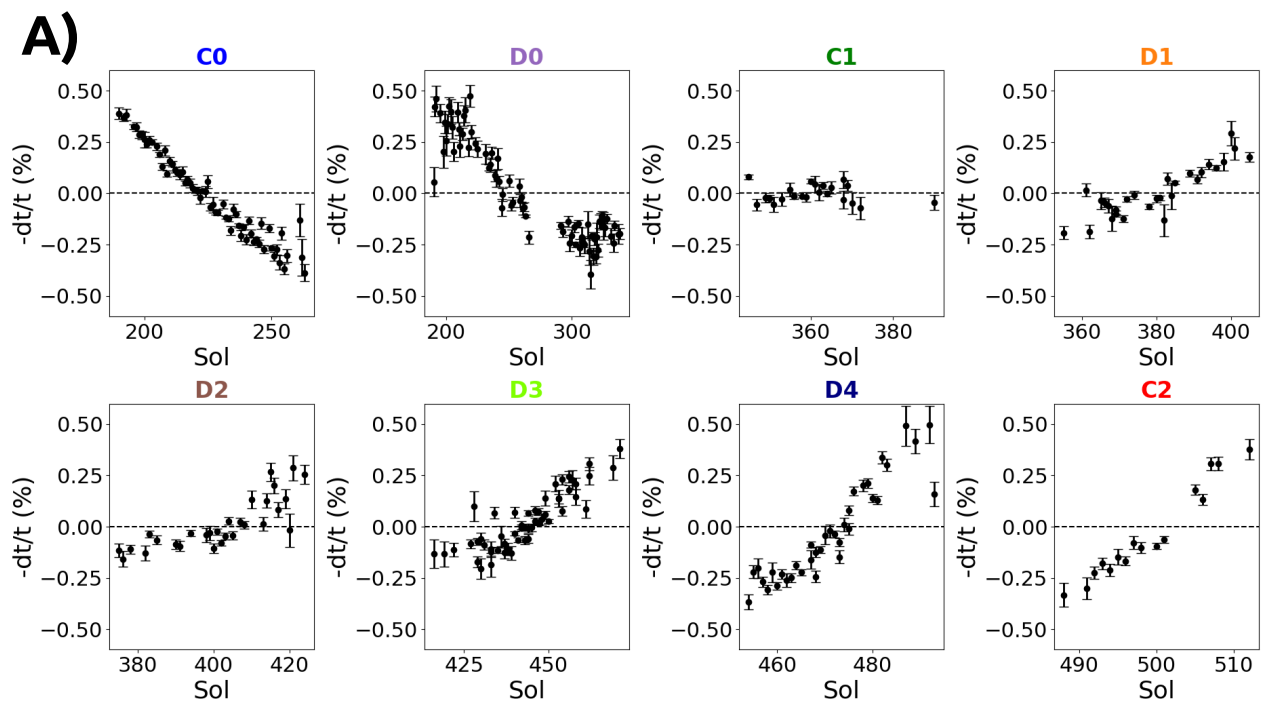

B)

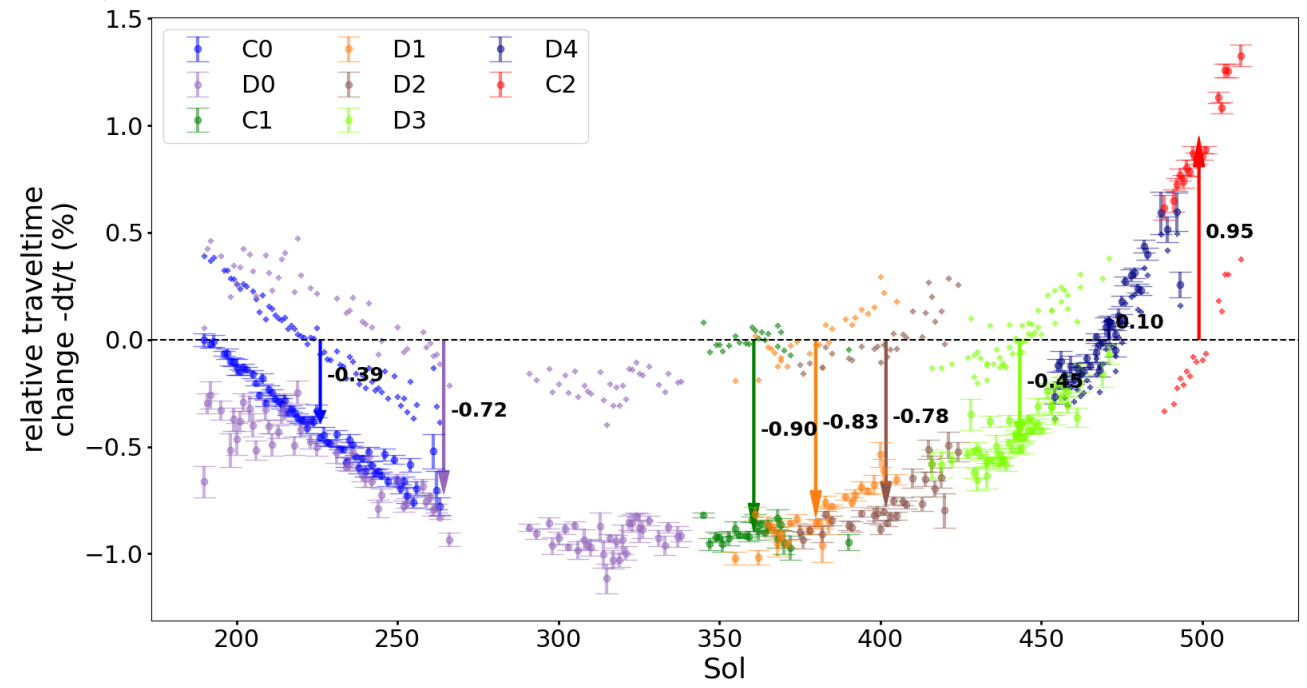

Figure 3. A) Measurements of the relative traveltime changes (-dt/t), in percent, in each family of SF events. Each waveform is compared to the average waveform of the corresponding family. B) Continuous traveltime changes curve obtained by combination of the relative measurements in each family in panel A. The color code of each family is the same as in Figure 1.

dom signal is equal to the inverse Fourier transform of its Power Spectral Density (PSD). It means that the information of temporal variations contained in an auto-correlogram is also contained in the corresponding spectrogram. As an example, in the context of structural health monitoring, the change of dominant frequencies of ambient noise spectrograms over time has been exploited to detect changes in the stiffness of structures during reinforcement works (Bottelin et al. 2017). Changes in ambient noise spectrograms are also expected in the case of seismic velocity changes. In the time domain, the effect of a homogeneous velocity variation on a seismic record $s(t)$ is a stretching of the waveform 
by a coefficient $\epsilon=d v / v=-d t / t$ which leads to a resulting waveform $s^{\prime}(t)=s(t \times(1+\epsilon))$. By Fourier transform we have in the frequency domain : $S^{\prime}(f)=\frac{1}{|1+\epsilon|} S\left(\frac{f}{1+\epsilon}\right)$ with $S(f)$ and $S^{\prime}(f)$ the Fourier transform of $s(t)$ and $s^{\prime}(t)$ respectively. Therefore, we see that a contraction of the timedomain Green's function due to a seismic velocity perturbation results in a stretching of its Fourier transform and vice-versa.

Using both numerical simulations and real data examples, Oren \& Nowack (2017) have suggested that the information on the arrival time of the various seismic phases contained in the Green's function (and thus partially contained in the empirical Green's functions reconstructed by autocorrelation) is encoded in the frequency domain in the form of rapid oscillations of the spectrum. In the case of multiply-scattered waveforms, such oscillations were in fact theoretically predicted by Shapiro (1986) and observed in laboratory experiments with ultrasound by Derode et al. (2001). In this setting, the typical separation between two maxima (or minima) of the PSD is known as the Thouless frequency which is proportional to the wave diffusivity in the medium. To enhance the visibility of the PSD oscillations, Oren \& Nowack (2017) suggest to deconvolve the original spectrum by a smoothed version of itself. This whitening operation in the spectral domain is classically knøwn in seismic interferometry as the deconvolution of the noise source function. Note that the spectral argument developed by Oren \& Nowack (2017) pertains to propagating waves and as such should also apply to seismic event records. To illustrate this point, we show in Figure 4.A the power spectral density (PSD) between 7 $\mathrm{Hz}$ and $9 \mathrm{~Hz}$ of all the SF events in the family C0 colored by Sol. We notice that all PSDs display similar oscillations, which constitute a sort of fingerprint of the signal. The frequency band structure visible on the spectrogram of Figure 4.B is therefore a consequence of the high similarity between the oscillations of each individual PSD. Furthermore, we remark on the spectrogram of Figure 4.B that the spectral oscillations show a clear drift in frequency with increasing Sols. This drift is the frequency domain signature of the stretching of the corresponding waveforms in Figure 1.B and agrees with the simple Fourier transform argument developed above. In what follows, we will show that a band structure, similar to the one obseryed on the seismic multiplets, can be identified in the high-frequency $(5-10 \mathrm{~Hz})$ background vibrations.

To uncover the frequency band structure, we apply the procedure of Oren \& Nowack (2017) to the data of the VBB sensors of SEIS. We perform the same pre-processing on the continuous records than for the event records in section 2.1. For each Sol, from Sol 180 to 866, we compute the power spectral density (PSD) of the 20 sample-per-seconds (sps) records in twenty-four time windows of one martian hour. The hourly PSD are computed using the Welch method with 12288 samples per segment (corresponding to 614.4 seconds at $20 \mathrm{sps}$ ) and an overlap of $70 \%$. This derived dataset enables two kinds of averaging. We may average the hourly PSD: 1) over several Sols to increase the signal-to- 


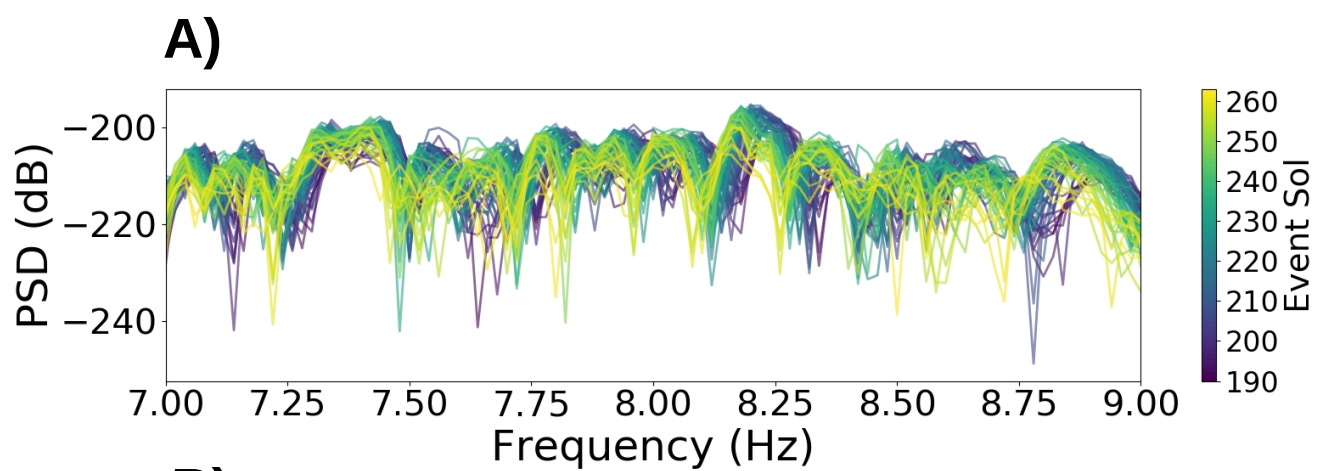

B)

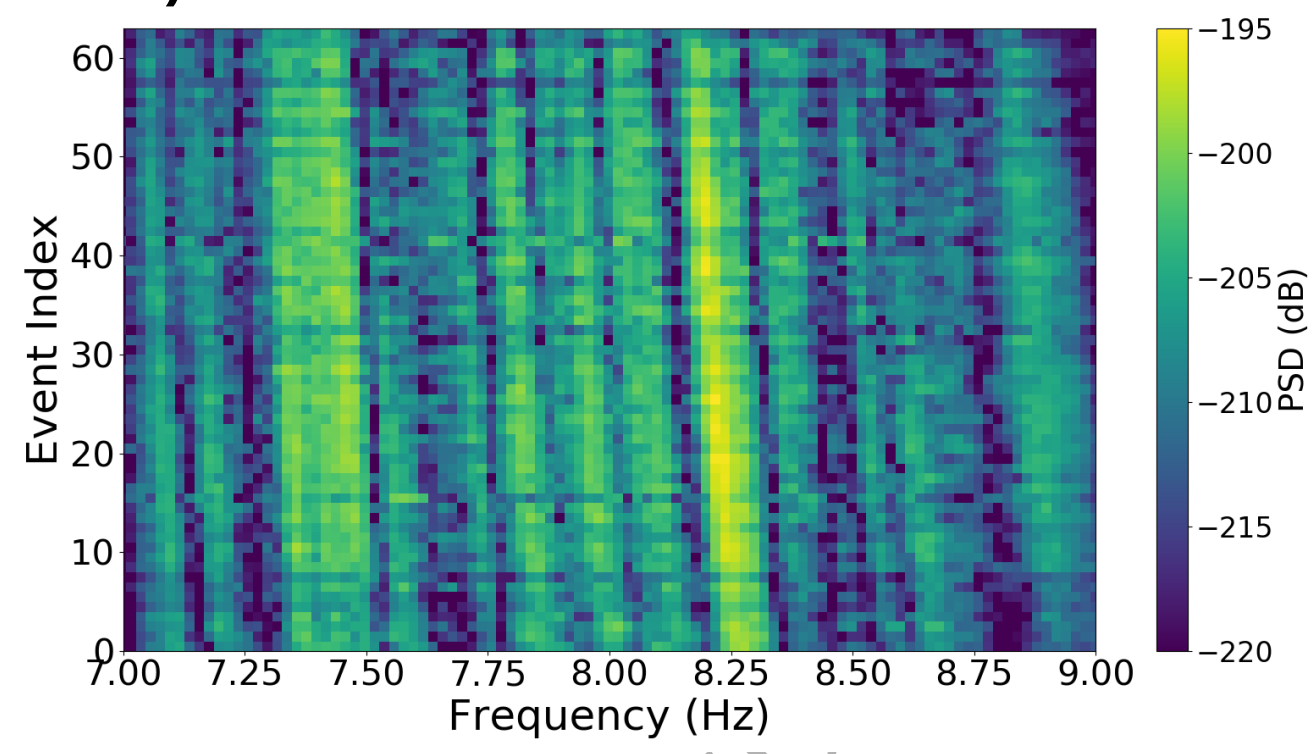

Figure 4. A) Power Spectral Density (PSD) in $\mathrm{dB}$ between $7 \mathrm{~Hz}$ and $9 \mathrm{~Hz}$ of the SF events of the family C0 colored by Sol. B) Spectrogram between $7 \mathrm{~Hz}$ and $9 \mathrm{~Hz}$ of the SF events of the family $\mathrm{C} 0$ ordered by increasing Sol.

noise ratio (SNR) at the daily scale or 2) over several martian hours to increase the SNR at the seasonal scale. Due to diurnal changes in the local atmospheric conditions, the daily record of SEIS can be divided in three distinct periods : a highly noisy daytime from 7h LMST (Local Mean Solar Time) to 17h LMST ; a low-noise evening from 18h LMST to 23h LMST and a mildly noisy morning from $0 \mathrm{~h}$ LMST to 6h LMST (Lognonn et al. 2020; Ceylan et al. 2021; Compaire et al. 2021). Therefore, when we perform averaging 2), we select only a subset of hourly PSDs in the most favorable time period. For instance, the Figure 5.A shows the PSD of the VBB North component during the evening period (18h to $23 \mathrm{~h}$ LMST) of Sol 350 . By applying this process to all Sols we obtain the spectrogram of the evening period shown in Figure 5.C. On this Figure we notice the broadband effect of the wind on the power level between Sol 500 and Sol 800. We also notice the narrow peaks of power corresponding to the well-identified lander modes around $1.6 \mathrm{~Hz}, 3.3 \mathrm{~Hz}, 4.1 \mathrm{~Hz}, 6.8 \mathrm{~Hz}$ and $8.6 \mathrm{~Hz}$ (Dahmen et al. 

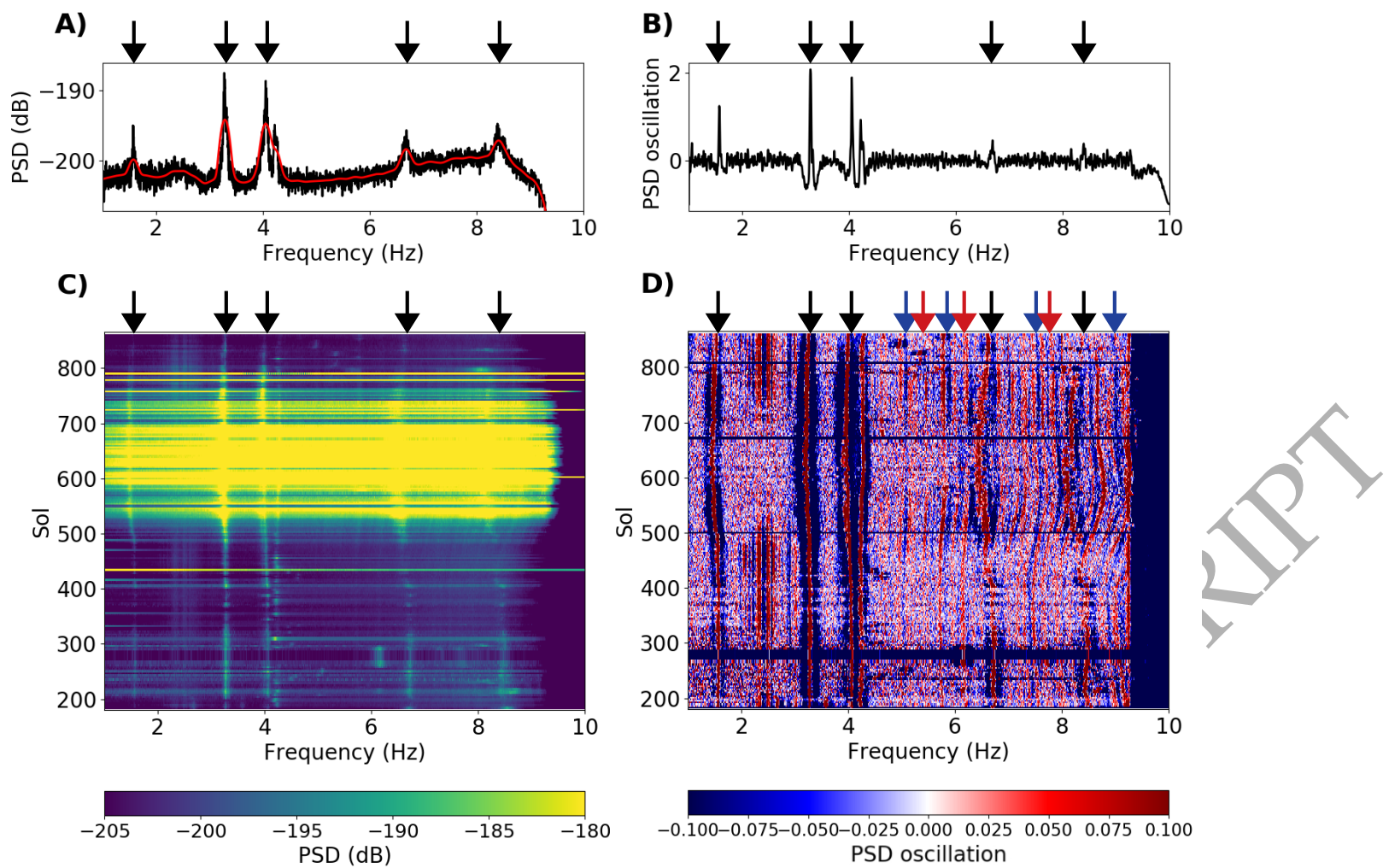

Figure 5. A) Power Spectral Density (PSD) in dB of the VBB North component between 18h and 23h LMST of Sol 350 (black curve) and its smoothed version (red curve) with a smoothing parameter of 0.32 Hz. B) Same PSD as in panel A after whitening and demeaning (PSD oscillation). C) Evening spectrogram of the VBB North component between the Sol 180 and Sol 866. D) Same spectrogram as in panel C after whitening and demeaning. The arrows indicate some positive (in red) and negative (in blue) lobes of the band structure. The black arrows at the top of each panel indicate the lander modes at $1.6 \mathrm{~Hz}, 3.3 \mathrm{~Hz}, 4.1 \mathrm{~Hz}, 6.8 \mathrm{~Hz}$ and $8.6 \mathrm{~Hz}$.

2021b). Between $2.2 \mathrm{~Hz}$ and $3 \mathrm{~Hz}$ we see the stable excitation of the so-called "2.4 $\mathrm{Hz}$ resonance" (Giardini et al. 2020; Ceylan et al. 2021; Driel et al. 2021; Compaire et al. 2021).

After averaging, we whiten the hourly PSD using a smoothing parameter of $0.32 \mathrm{~Hz}$ chosen empirically and remove the mean from the whitened PSD to obtain what we will call in the following the "PSD oscillations". In Figure 5.A the smoothed PSD is plotted in red. The corresponding PSD oscillation is shown in Figure 5.B. The whitened and demeaned version of the spectrogram in Figure 5.C is shown in Figure 5.D. In addition to the features mentioned previously, this treatment reveals a remarkable oscillation in the spectrogram above $5 \mathrm{~Hz}$ that we can follow from one Sol to the next. In the following we will refer to this feature using the designation "band structure". This band structure is observed only on the horizontal components (North and East) and not on the vertical component (see Figure A4 in B). The second remarkable observation is that the band structure presents a strong seasonal variation with a pattern that differs from the lander modes in the 1-9 $\mathrm{Hz}$ band. 
When we investigate the PSDs oscillations at a higher time resolution the differences between the lander modes spectra and the band structure in terms of temporal variation show up even more clearly. In Figure 6 we represent the hourly PSD oscillation of the North component from the beginning of Sol 350 to the end of Sol 352. In this figure we can observe the daily variations of the lander modes eigenfrequencies at $1.6 \mathrm{~Hz}, 3.3 \mathrm{~Hz}, 4.1 \mathrm{~Hz}, 6.8 \mathrm{~Hz}$, and $8.6 \mathrm{~Hz}$ reported by several articles (Lognonn et al. 2020; Giardini et al. 2020; Ceylan et al. 2021; Driel et al. 2021; Compaire et al. 2021). We also remark that the higher the eigenfrequency of a given lander mode, the more pronounced is its absolute variation during a Sol. With a time resolution of one hour, it is difficult to follow frequency variations that are too large and too fast. In particular the $8.6 \mathrm{~Hz}$ mode goes from a resonance frequency of 8.6 $\mathrm{Hz}$ to about $7 \mathrm{~Hz}$ in only 2 hours (during sunrise), and inversely during sunset (Dahmen et al. 2021b). In Figure 6, the $8.6 \mathrm{~Hz}$ mode is only clearly visible during morning time. We also observe that the 2.4 $\mathrm{Hz}$ resonance is only observable during the evening, when the disturbances induced by the atmosphere are the lowest as noted by Compaire et al. (2021).

Figure 6 shows that the oscillations in the frequency content above $5 \mathrm{~Hz}$ are also recovered at a temporal resolution of one hour. The band structure does not seem to vary with local time, unlike the lander modes, but the highly energetic $6.8 \mathrm{~Hz}$ and $8.6 \mathrm{~Hz}$ lander modes hamper the detection of the band structure as their eigenfrequency is modulated by the local temperature. Therefore nighttime is the best period to study the variability of the band structure over long periods of time (several tens of Sols) as it is the period where the $6.8 \mathrm{~Hz}$ and $8.6 \mathrm{~Hz}$ lander modes are the most stable and well-localized. In section 3.2, we develop a signal processing workflow to enhance the visibility of the band structure and subsequently show that relative traveltime changes deduced from these data are consistent with the measurements performed on SF events waveforms. Before going into details of the processing, we would like to emphasize that the non-stationarity of lander modes at daily to seasonal time-scales makes it inconvenient to look for waveform variations using auto-correlograms in the time-domain. We could of course pre-process the data using e.g. notch filters to mute the lander modes. However, to be efficient this procedure would require the application of a time-dependent filter whose properties would evolve on seasonal scales (to adapt to the Lander modes). This would artificially create waveform with time-dependent properties that could hamper the detection of genuine variations induced by changes in the medium properties.

\subsection{Temporal variations}

To be specific, we point out that what we call a "band" in the band structure is a local extremum of the PSDs that can be tracked from one Sol to the next. With the whitening and demeaning procedure described in section 3.1, these extrema alternate from negative (blue bands) to positive (red bands) 


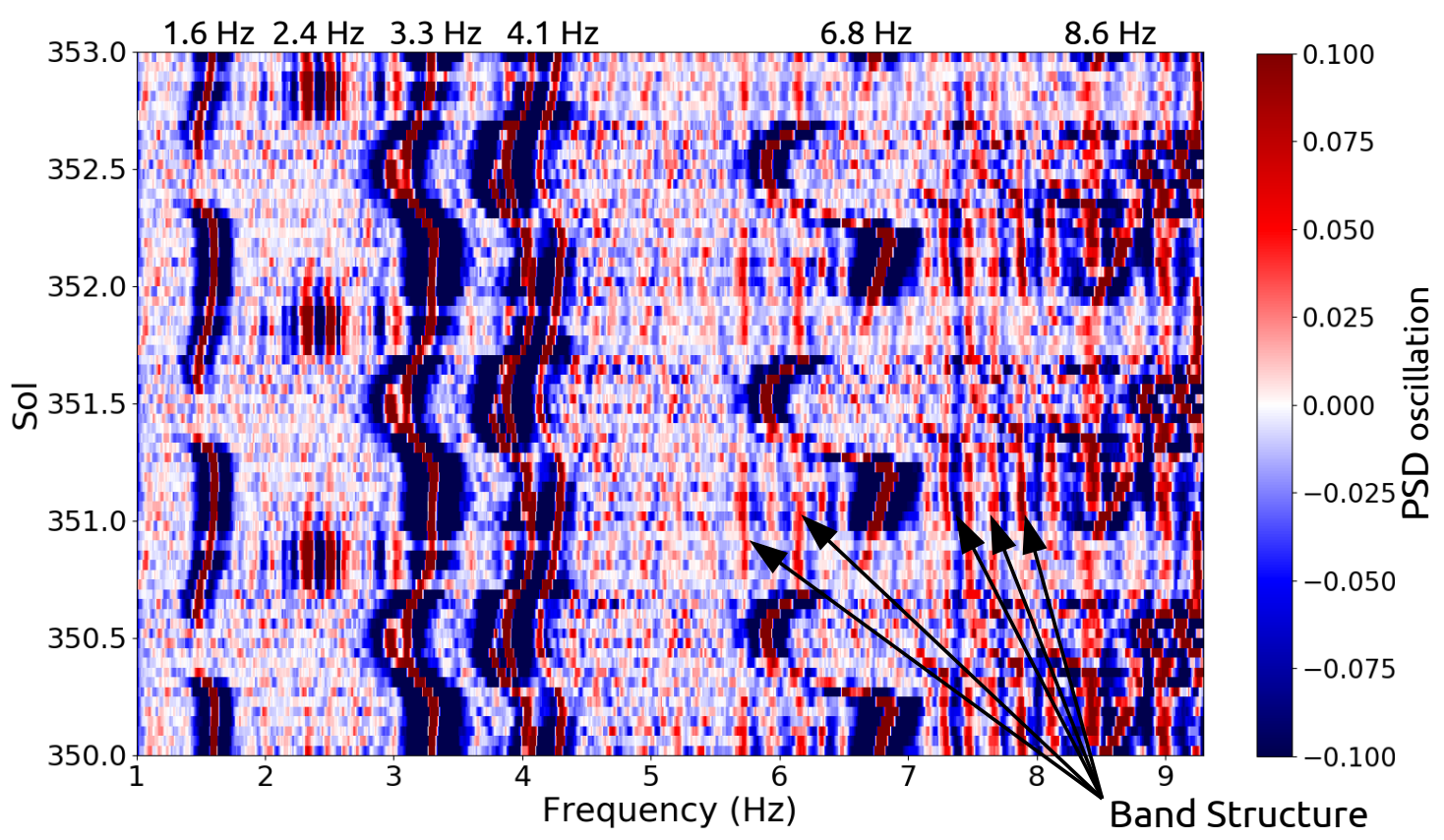

Figure 6. Hourly PSD oscillation of the VBB North component between Sol 350-0h LMST to Sol 352-24h LMST. On the vertical axis the martian hours (LMST) are counted by fraction of Sol (for instance 350.5 correspond to Sol 350-12h LMST). The name of the six features discussed in the text of section 3.1 are indicated at the top. From left to right we have the lander modes at $1.6 \mathrm{~Hz}$, the $2.4 \mathrm{~Hz}$ resonance, the lander mode at $3.3 \mathrm{~Hz}$, a doublet structure corresponding to the lander mode at $4.1 \mathrm{~Hz}$, the lander mode at $6.8 \mathrm{~Hz}$ and the lander mode at $8.6 \mathrm{~Hz}$. This last one is only observable during the morning period in this range of Sol.

(see Figures 5.D and 6). Having identified a local extremum, we now develop a procedure to follow its location throughout the Martian year.

\subsubsection{Noise reduction}

To facilitate the band tracking over a full martian year, we applied a noise reduction method inspired by Moreau et al. (2017), which applies to the matrix of the evening PSD oscillations. The $(i, j)$ matrix element corresponds to the PSD at Sol $i$ and frequency $f^{j}$. The SVD-based Wiener filter (SVDWF) described in Moreau et al. (2017) is a three steps method. First, a given corrupted 2D-matrix (signal with noise) is decomposed into singular eigenvectors of which only a fraction is preserved (SVD filtering). Second, a Wiener filter is applied to each individual singular eigenvector before recombining them to build a de-noised matrix. Finally, a Wiener filter is applied to the reconstructed matrix from the previous step. In 2D, a Wiener filter is described by two parameters : $K$ and $L$. The parameter $K$ corresponds to the length of the smoothing window in the vertical dimension of the matrix and 
the parameter $L$ to the length of the smoothing window in the horizontal dimension. In our case the horizontal dimension corresponds to the frequency and the vertical dimension to the mission time-line.

We apply a SVD filtering to the matrix represented in Figure 7.A. We keep 6\% of the singular vectors, which correspond to $30 \%$ of the total variability. We do not apply Wiener filtering to each singular vector individually as in the procedure of Moreau et al. (2017). However, we do apply a Wiener filter to the recomposed matrix after SVD filtering. We adjust $L$ and $K$ in order to apply a smoothing of $0.05 \mathrm{~Hz}$ along the frequency dimension and of 5 Sols along the time-line dimension. The resulting matrix of the evening PSD oscillation after the denoising procedure is shown in Figure 7.B. The visibility of the band structure is clearly enhanced by the combined SVD+Wiener filtering.

\subsubsection{Band tracking}

After successful denoising of the original PSD matrix, we perform band tracking using a correlation method. We work in sliding time-line windows of $N$ Sols duration with $25 \%$ overlap and use an index $k$ to label the time windows. $M$ hourly PSDs are evaluated for each SOL. We tested our algorithm on both the full day $(M=24)$ or from $18 \mathrm{~h} 00$ to $23 \mathrm{~h} 00 \mathrm{LMST}(M=5)$ and did not find significant differences bewteen the two. Note that the first time-line window starts on Sol 185 (00h00 LMST). The tracking process is initialized at frequency $f_{k=0}^{i}$ and time $t_{k=0}^{i}$, the latter referring to the central time of the initial time-line window. As an example for $N=5 M=24$, one has $t_{0}^{i}=12 \mathrm{~h} 00 \mathrm{LMST}$ on Sol 187. We then compute cross-correlation functions between the PSD oscillations of time windows $t_{k}^{i}$ and $t_{k}^{i}+1$, restricted to the frequency band $\left[f_{0}^{i}-\alpha . f_{0}^{i} ; f_{0}^{i}+\alpha . f_{0}^{i}\right]$. The parameter $\alpha$, which controls the bandwidth, is adjusted in a way such that the frequency band is sufficiently large to contain several oscillations of the PSD, yet sufficiently narrow to avoid contamination by lander modes. The first, second, $\cdots,(N \times M)^{\text {th }}$ PSD of window 0 is correlated, respectively, with the first, second, $\cdots$, $(N \times M)^{\text {th }}$ PSD of window $k$. At the end of the process we obtain $N \times M$ cross-correlation functions, all restricted to the same frequency band. Each cross-correlation function has a maximum at a given frequency shift. In time-line window $k$, the position in frequency of the band initialized at $f_{0}^{i}$ is given by $f_{k}^{i}=f_{0}^{i}+\Delta f_{k}^{i}$, where $\Delta f_{k}^{i}$ is defined as the average of the $N \times M$ frequency shifts measured by cross-correlations between windows 0 and $k$. The associated error, $\sigma_{k}^{i}$, is the standard deviation (std) of the $N \times M$ frequency shifts.

Note that the bands visible in Figure 7.B have a bandwidth of the order of $0.1 \mathrm{~Hz}$. Several frequencies can thus be chosen for the initialization of a given band depending on the resolution of the frequency vector. In figure 7.C we show the results of the band tracking procedure for 8 initial frequencies below the $6.8 \mathrm{~Hz}$ mode and 8 initial frequencies above (black points) with $N=5$ Sols, $M=24$ hours and $\alpha=0.03$. We then proceed to a selection based on the standard deviations to keep 
only the measurements with a relative error lower than $0.7 \%$. All the consecutive points with a relative variation higher than $0.1 \%$ are considered as outliers and therefore removed. The measurements that fulfill all selection criteria are shown in Figure 7.D.

\subsubsection{Relative traveltime changes}

To measure the relative traveltime changes from the temporal variations of the frequency band structure, we exploit the time-frequency duality of the Fourier transform. Indeed, a stretching coefficient $\epsilon=-d t / t$ applied to a time domain signal results in a shift $d f / f$ of its band structure. For each sequence of frequencies $\left(f_{k}^{i}\right)_{k}$ described in the previous section we construct a sequence of stretching coefficients $\left(\epsilon_{k}^{i}\right)_{k}$ relative to the initial frequency $f_{0}^{i}$ as $\epsilon_{k}^{i}=\frac{f_{k+1}^{i}-f_{0}^{i}}{f_{0}^{i}}$, at times $t_{k+1}^{i}$ and with the associated error $E_{k}^{i}=\frac{\sigma_{k+1}^{i}}{f_{k+1}^{i}}$.

To reduce the number of gaps in the sequences due to the selection procedure and mitigate the potential tracking errors, we combine together the 8 sequences of stretching coefficients measured below the $6.8 \mathrm{~Hz}$ mode and the 8 sequences measured above. The combination is performed by averaging both the stretching coefficients and their associated errors. In Figure 7.E we show the two resulting sequences of relative traveltime changes in black for the measurements made between $5.3 \mathrm{~Hz}$ and 6.2 $\mathrm{Hz}$, and in blue for the measurements made between $7.1 \mathrm{~Hz}$ and $8.0 \mathrm{~Hz}$. We also report in Figure 7.E the continuous sequence of $-d t / t$ coming from the SF events analysis (section 2.2).

The key observation in Figure 7.E is the very good agreement between the relative traveltime changes measured in the SF events and in the band structure of the background vibrations. Both the amplitude of the variations and the trend are consistent between the two datasets. Figure 7.E also confirms the observations from the Family $\mathrm{C} 0$ in Figure 2.D, i.e. the traveltime changes are highly frequency dependent. In particular the trend evolves in the same direction when the observation frequency varies (the lower the frequency, the lower the local slopes of the variation). This observation strongly suggests an identical origin for the relative traveltime/frequency changes observed in both SF events and background vibrations.

\section{THERMOELASTIC MODEL}

In this section, we propose a simple interpretation of our observations based on the thermoelastic response of the subsurface (Berger 1975). 
A)

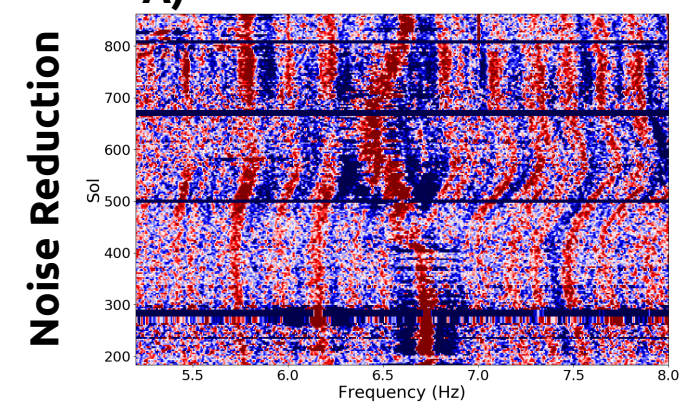

C)

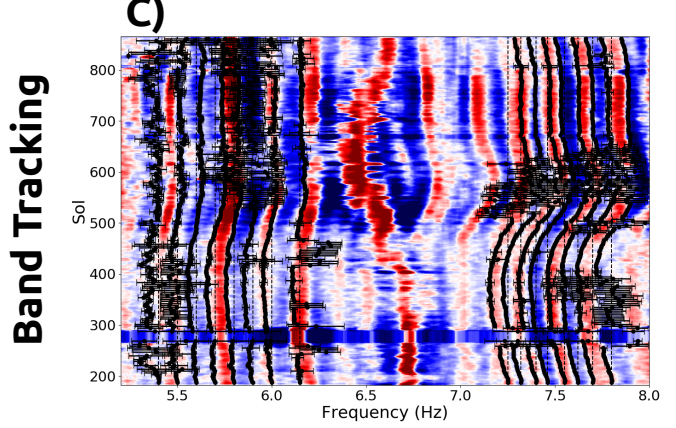

B)

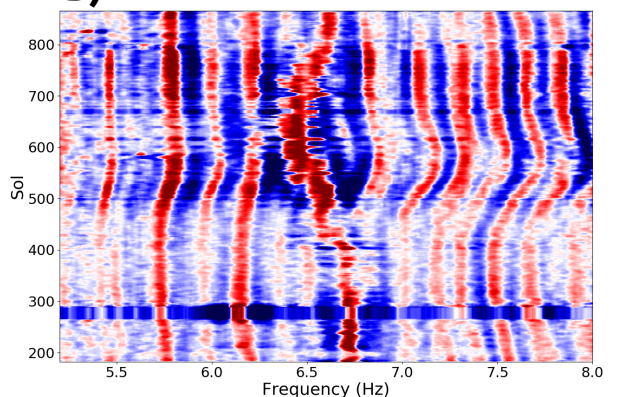

D)

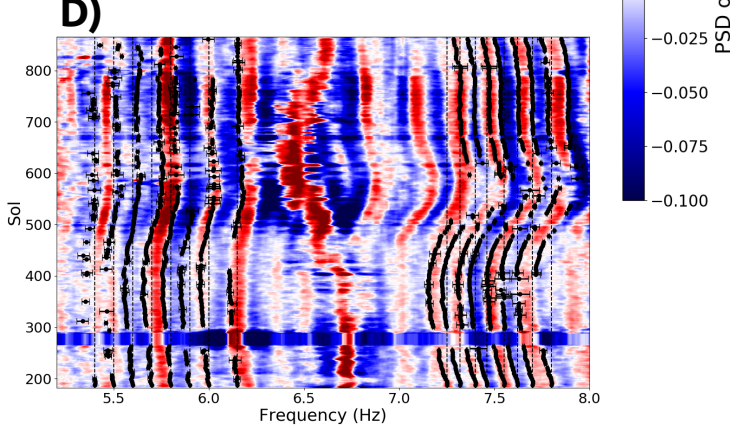

E)

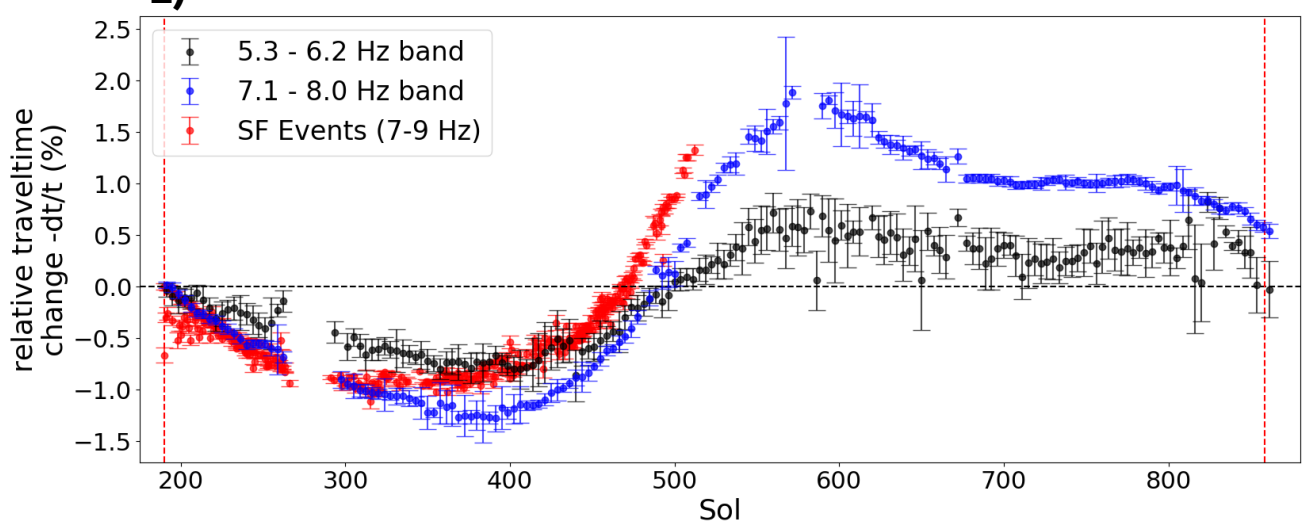

Figure 7. A) Oscillation of the Evening PSD of the VBB North component from Sol 181 to Sol 866 in the frequency band 5.3-8.0 Hz. At the center of the frequency band we see the lander mode at $6.8 \mathrm{~Hz}$ varying seasonally with a trend that differ from the band structure. B) Same as in panel A but after the application of the denoising procedure inspired by Moreau et al. (2017). C) Results of the band tracking procedure described in section 3.2 for 16 initial frequencies $(5.40 \mathrm{HZ}, 5.50 \mathrm{~Hz}, 5.60 \mathrm{~Hz}, 5.70 \mathrm{~Hz}, 5.80 \mathrm{~Hz}, 5.90 \mathrm{~Hz}, 6.00 \mathrm{~Hz}, 6.15$ $\mathrm{Hz}, 7.25 \mathrm{~Hz}, 7.32 \mathrm{~Hz}, 7.40 \mathrm{~Hz}, 7.46 \mathrm{~Hz}, 7.55 \mathrm{~Hz}, 7.62 \mathrm{~Hz}, 7.70 \mathrm{~Hz}, 7.80 \mathrm{~Hz}$ ), superimposed on the spectrogram of panel B. D) Same as in panel C after the selection procedure. E) Relative traveltime changes derived from the frequency variations measured in the band structure in the 5.3-6.2 Hz frequency band (black points) and in the 7.1-8.0 Hz frequency band (blue points). The red points correspond to the continuous sequence of relative tráveltime changes derived from the SF events in section 2.2. The two vertical red dashed lines are spaced of one mártian year (668.6 Sols). 


\subsection{Thermal stresses and implications for seismic velocities}

Thermoelasticity has previously been put forward as the dominant mechanism to explain seasonal subsurface velocity variations in dry granular media on Earth (Richter et al. 2014). Berger (1975) has shown that a non-uniform distribution of temperature at the surface of an elastic medium is at the origin of thermal stresses at depth. He imagined a surface temperature forcing of the form:

$$
T(x, z=0, t)=T_{m}+\sum_{\omega} T_{\omega} \cos \left(k_{x} x\right) \cos \left(\omega t+\phi_{\omega}\right)
$$

where the sum runs over the characteristic pulsations $\omega$ and the following notations have been introduced: $T_{\omega}$ and $\phi_{\omega}$ are, respectively, the characteristic amplitudes and phases of the surface temperature perturbation (at pulsation $\omega$ ) and $T_{m}$ its average value. In Eq. (2), each mode generates a heat wave which propagates at depth with a characteristic attenuation length, also called skin depth, $1 / \gamma=\sqrt{2 D / \omega}$ where $D=\kappa / \rho C_{p}$ is the thermal diffusivity of the medium. Here, $\kappa, \rho$ and $C_{p}$ are respectively the thermal conductivity, the density and the specific heat capacity. As the temperature propagates into the ground, it acquires a phase delay $\gamma z$ with respect to the surface value $\omega t+\phi_{\omega}(z$ is the depth). Note that for realistic values of the diffusivity, the seasonal heat wave is not expected to penetrate deeper than 2 to 3 meters under InSight (Siegler et al. 2017).

A key ingredient of the thermoelastic model is the modulation of the temperature in direction $x$ along the surface with characteristic wavelength $l_{x}=2 \pi / k_{x}$. Such a dependence may be justified by invoking the effects of topography or geological heterogeneity around InSight. Whatever the origin of the lateral variations, we follow Berger (1975) and assume for simplicity that they may be modeled adequately by imposing a non-uniform surface value for the temperature, characterized by a single wavelength. Solving the elasticity equations, Berger (1975) shows that the associated non-uniform thermal dilation of the material (with expansion coefficient $\alpha$ ) associated with the propagation of the thermal wave at depth generates elastic stresses at depth which, in turn, perturb locally the seismic wavespeeds (Richter et al. 2014):

$$
\begin{array}{r}
\frac{\Delta v}{v}(x, z, t)=-2 \alpha b \frac{\partial \rho v^{2}}{\partial \sigma} \sum_{\omega} T_{\omega} \cos \left(k_{x} x\right)\left[\cos \left(\omega t+\phi_{\omega}-\gamma z\right) e^{-\gamma z}\right. \\
\left.-\frac{(1+\nu)}{\sqrt{2 \gamma^{2}+2 \gamma k_{x}+k_{x}^{2}}} \cos \left(\omega t+\phi_{\omega}-\psi\right) k_{x} e^{-k_{x} z}\right],
\end{array}
$$

where the phase $\psi$ is defined by:

$$
\tan \psi=\frac{\gamma}{\gamma+k_{x}} \text { with } \psi \in[0, \pi / 2[.
$$

The notations introduced in Eq. (3) are as follows: 
$v$, seismic velocity ( $P$ or $S$ ), $\alpha$, thermal expansion coefficients, $\sigma$, principal thermal stress and $\nu$, Poisson's ratio. The parameter $b$ depends on the wavetype, as recalled hereafter:

$$
b= \begin{cases}\frac{(1+\nu)(1-2 \nu)}{2(1-\nu)^{2}} & \text { for P waves } \\ \frac{1+\nu}{1-\nu} & \text { for } \mathrm{S} \text { waves }\end{cases}
$$

Further computational details are provided in D. Since the model neglects lateral transfer of heat, the validity of the result (3) is limited to the regime $\gamma \gg k_{x}$. In other words, in the horizontal direction, the temperature must vary slowly at the scale of the penetration depth of the heat wave $1 / \gamma$. In this case, one finds that $\psi \approx \pi / 4$, in agreement with Richter et al. (2014).

Qualitatively, the first term of Eq. (3) informs us that in a region where the temperature is higher (resp. lower) than in its surrounding, the material dilation (resp. contraction) is restrained and the thermal stress is compressive (resp. extensive), which results in an increase (resp. decrease) of the velocity. This term is entirely controlled by the propagation of the heat wave at depth and therefore attenuates at the scale $1 / \gamma$. For depths greater than a few temperature skin depths, the second term of Eq. (3) takes over. It may be understood as the elastic response at depth induced by the surface temperature modulation. While the amplitude of this term is small (as compared to the one of the first term), it induces perturbations at a much larger scale $l_{x}$ and its integral over depth is of order 1 . As a consequence, in spite of its small magnitude, this term may not always be negligible. This point will be further discussed in the sequel.

\subsection{Application to the case of study}

To make a quantitative comparison between the thermoelastic model and the observations of apparent velocity variations, the characteristic pulsations, amplitudes, and phases of the temperature forcing have been determined. The surface temperature shown in Figure 8.A has been calculated from a numerical model of temperature propagation into the subsurface using the incident solar flux deduced from the JPL Horizon Ephemeris as forcing. In this model, the surface temperature results from a balance between the incident, radiative and conductive energy fluxes. In the model, the thermal properties are uniform with conductivity $\kappa=0.039 \mathrm{~W} / \mathrm{m} / \mathrm{K}$ Grott et al. (2021), $\rho=1800 \mathrm{~kg} / \mathrm{m}^{3}$ and $C_{p}=600$ $\mathrm{J} / \mathrm{kg} / \mathrm{K}$. These values are consistent with those derived from the observed daily surface temperature fluctuations observed by the T2 radiometer of InSight Piqueux et al. (2021); Mueller et al. (2021). More information on the temperature data used in this study are given in C. At the seasonal time scale, the model provides a decent approximation to the observed temperatures but cannot capture temperature fluctuations related to the local meteorology such as dust storms that opacify the atmosphere. The 
modeled variations of the temperature at depth are represented as a function of time (expressed in Sol since the landing of the InSight mission) in Figure 8.B.

To establish the link between surface temperature and seismic velocity variations, we introduce a simple seismological model for SF events. The lack of coherent arrivals and the emergent character of the signal suggests that SF waveforms are composed of multiply scattered waves. As recalled in section 2.1, the strong correlation between the local temperature and the triggering of these events also favors the idea that they are excited by local and shallow seismic sources (Dahmen et al. 2021a). Consistent with these observations, we propose to model SF events as Rayleigh waves scattered by the near surface heterogeneities and interpret the traveltime perturbation in the coda as the result of a perturbation in the Rayleigh wave phase velocity. The decent agreement between $d v / v$ inferred from $\mathrm{SF}$ events and the frequency band structure suggests that Rayleigh waves also dominate the noise wavefield. Rayleigh wave dominance will be our working assumption in the rest of the paper.

Thanks to the literature compilation found in Richter et al. (2014), we can make reasonable assumptions on the values of acoustoelastic constants to convert the thermal stress model to a profile of seismic velocities perturbations. For the partial derivatives of $\mathrm{P}$ and $\mathrm{S}$ wavespeeds $v_{p}, v_{s}$ with respect to stress, we assume $\partial \rho v_{p}^{2} / \partial \sigma=\partial \rho v_{s}^{2} / \partial \sigma=-700$. The negative sign of the acousto-elastic constants corresponds to the seismological definition of positive (respectively negative) elastic stress $\sigma$ for extensional (respectively compressional) deformation (see also Winkler \& McGowan 2004). In absolute value, the typical range of acoustoelastic constants provided by Richter et al. (2014) is 2001000 for uniaxial stress and 500-5000 for hydrostatic stress. Additional parameters of the model are fixed as follows: thermal expansion coefficients $\alpha=3 \times 10^{-5} \mathrm{~K}^{-1}$, Poisson's ratio $\nu=0.2$. For the wavenumber of the surface temperature modulation $k_{x}=2 \pi / l_{x} \mathrm{~m}^{-1}$ we choose the ad-hoc value $l_{x}=1000 \mathrm{~m}$, for which the first term in the solution (3) is largely dominant over the second. Considering an average shear wave speed in the subsurface of $100 \mathrm{~m} / \mathrm{s}$ and $v_{p} / v_{s}=1.63$, this corresponds to the regime $l_{x} \gg \lambda_{r}$ with $\lambda_{r}$ the Rayleigh wavelength. The case where $\lambda_{r}$ and $l_{x}$ are of the same order will be discussed later.

The perturbation of the seismic velocity field at depth corresponding to the temperature variation shown in Figure 8.B is represented in Figure 8.C. We recall that the perturbation of the seismic velocity field is the result of the difference of two terms. The first term follows the thermal perturbation. It decreases as $e^{-\gamma z}$ and is delayed with respect to the surface forcing with a phase $\gamma z$. The second term decreases as $e^{-k_{x} z}$ and exhibits a constant phase shift with respect to the surface temperature. In Figure 8.C we see that the first term dominates in the first three meters. At four meters depth the second term begins to offset the first term and below six meters depth the second term dominates entirely. Qualitatively, when $l_{x}$ is large with respect to the skin depth $1 / \gamma$ (which is the case of Figure 
8.C), the amplitude of the second term is small compared to the one of the first term but its influence extends at much greater depth. As a consequence, the effect of the thermoelastic perturbation on the apparent velocity change in the coda of seismic events or background vibrations is highly dependent on the depth sensitivity of Rayleigh waves to perturbations in $P$ and $S$ seismic velocities.

To estimate the Rayleigh wave phase velocity perturbations, we employ the sensitivity kernels derived in Aki \& Richards (2002). Examples of phase velocity sensitivity kernels are superimposed on Figure 8.C for $\mathrm{P}$ and $\mathrm{S}$ velocity perturbations at two different frequencies $(6 \mathrm{~Hz}$ in green and $8 \mathrm{~Hz}$ in yellow). We see that the sensitivity to P-wave speed perturbations is limited to the shallow subsurface $(z<3 \mathrm{~m})$, where the perturbations are in fact largest. This behavior can be traced back to the strongly evanescent nature of the P-wave contribution to the Rayleigh mode which decays with a characteristic scale length $f^{-1} \times\left(1 / c_{r}^{2}-1 / c_{p}^{2}\right)^{-1 / 2}\left(f\right.$, the wave frequency, $c_{r}$ the Rayleigh wavespeed, $c_{p}$, the longitudinal wavespeed) equal to $1.5 \mathrm{~m}$ (resp. $1.1 \mathrm{~m}$ ) at $f=6 \mathrm{~Hz}$ (resp. $f=8 \mathrm{~Hz}$ ). By contrast, the sensitivity to $\mathrm{S}$-wave speed perturbations displays a maximum at depth, below which it decreases with a characteristic scale-length $f^{-1} \times\left(1 / c_{r}^{2}-1 / c_{s}^{2}\right)^{-1 / 2}\left(c_{s}\right.$, the shear wavespeed) equal to $37 \mathrm{~m}$ (resp. $26 \mathrm{~m}$ ) at $6 \mathrm{~Hz}$ (resp. $8 \mathrm{~Hz}$ ). Although the largest velocity perturbations are found in the first two meters below the surface, the shear wavespeed kernel makes the Rayleigh wave phase velocity sensitive to changes in the principal stress field up to at least 25 meters in the frequency band of study.

In Figure 9.A, we represent on the same plot the modeled seasonal temperature variation at the surface and the apparent relative velocity changes measured in both ambient noise (North component) and SF events (East component). The strong correlation between the temperature and velocity time series is striking. A comparison between the observed apparent velocity changes and the predictions of the thermoelastic model is shown in Figure 9.B at two different seismic frequencies $(f=6 \mathrm{~Hz}$ in black line and $f=8 \mathrm{~Hz}$ in blue line). Our simple model reproduces quite well the main features of the seasonal variations of velocity detected in the high-frequency seismic data. We note in particular that thermo-elasticity introduces a notable time delay of about 50 Sols between the surface temperature signal and the apparent velocity change. This property can be traced back to the slow propagation of the surface temperature signal in the weakly conductive regolith. The asymmetry between the positive and negative velocity perturbations is also decently reproduced by the model as well as the weak frequency dependence of the time delay between temperature and seismic velocity changes.

Probably the least-well reproduced feature of the observed $d v / v$ variations is the frequency dependence of their amplitude. The strong increase of $d v / v$ between the $5.3-6.2 \mathrm{~Hz}$ and $7.1-8.0 \mathrm{~Hz}$ frequency band is clearly missed. We verified that this discrepancy cannot be improved by changing the wavelength $\left(l_{x}\right)$ of the temperature modulation along the surface. When $l_{x}$ is large with respect to the Rayleigh wavelength, the thermoelastic stress is largely dominated by the restrained dilation- 


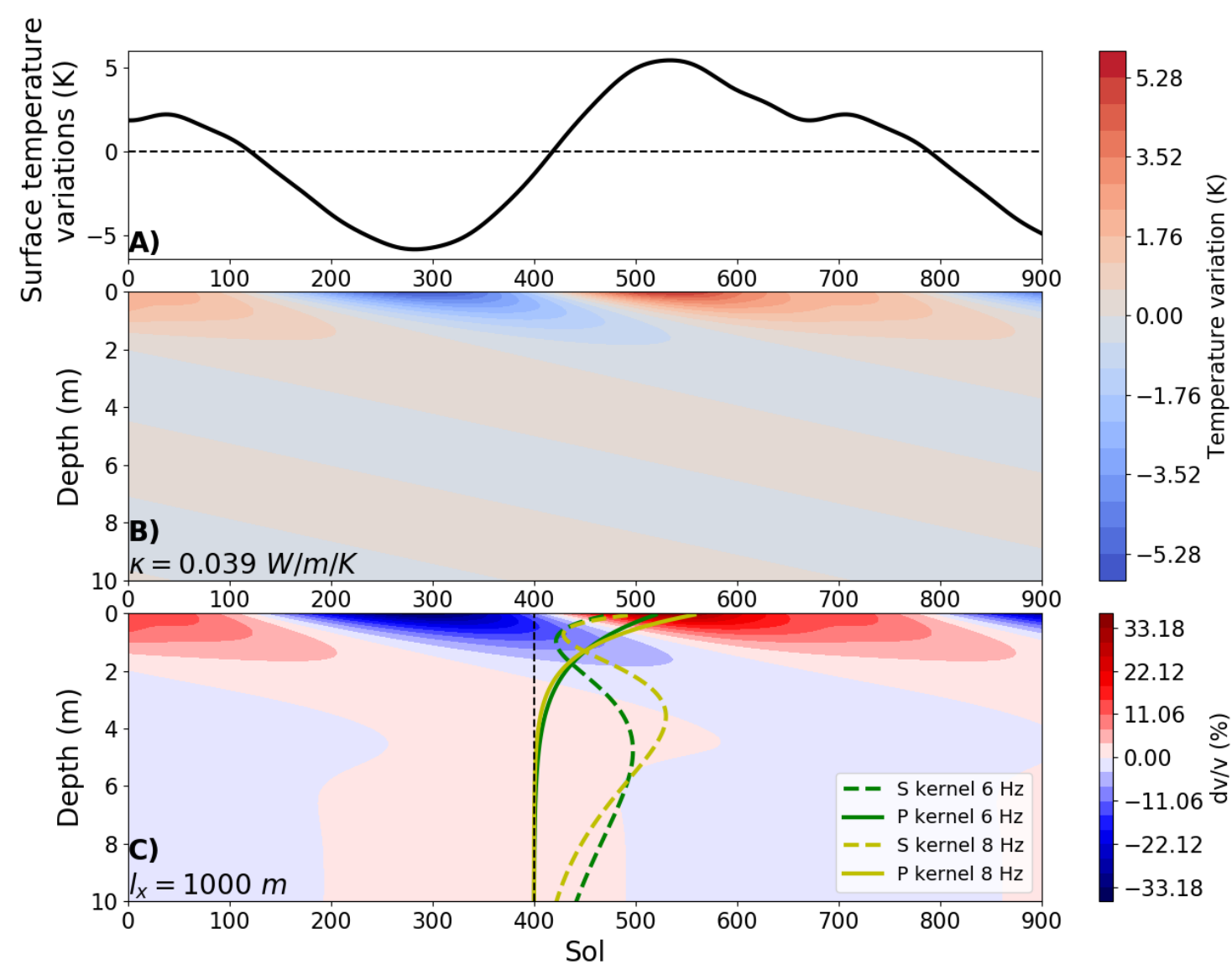

Figure 8. A) Model of surface temperature in Kelvin calculated from the incident solar flux deduced from the JPL Horizon Ephemeris. B) Variation of the temperature field in depth according to the time in Sol due to the variation of the incident solar flux considering a thermal conductivity $\kappa=0.039 \mathrm{~W} / \mathrm{m} / \mathrm{K}$. C) Relative velocity variation (\%) in depth induced by the temperature field perturbation shown in (B) as predicted by the thermoelastic model (Eq. (3)) with the parameters given in the text of section 4.2 and with the $b$ parameter of $\mathrm{S}$-waves (see Eq. (5)). Superimposed to the velocity variation, we show the sensitivity kernels for P-waves (solid lines) and S-waves (dot lines) at $6 \mathrm{~Hz}$ (green) and $8 \mathrm{~Hz}$ (yellow).

contraction of the material controlled by the temperature propagation at depth (term in $e^{-\gamma z}$ ), which is independent of $l_{x}$. It means that all the values of $l_{x}$ above 1000 meters (used in Figure 8.C and Figure 9.B) will all yield practically the same time delays between $d v / v$ and the surface temperature. In the next section, we discuss the impact of $l_{x}$ on our results and propose a simple mechanism to explain the frequency dependent $d v / v$ amplitude. 

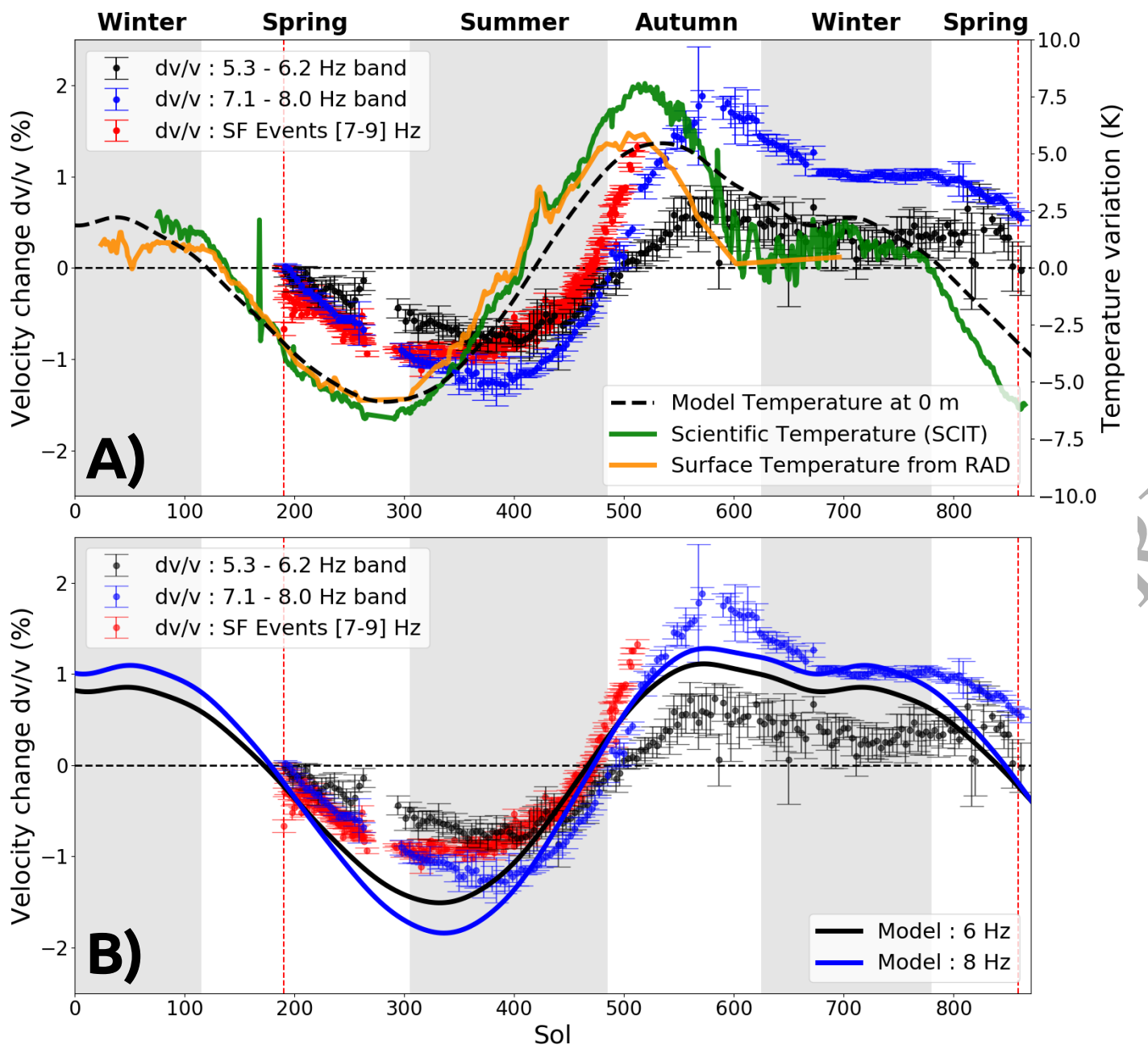

Figure 9. A) Comparison between the observed velocity variations in SF events (red points) using the East component, background vibrations in two different frequency bands ([5.3-6.2] $\mathrm{Hz}$ in black and [7.1-8.0] Hz in blue) using the North component and the daily average surface temperature. The orange line is the daily average surface temperature measured by the radiometer RAD Mueller et al. (2020). The green line is the daily average Scientific Temperature of SEIS instrument (see C). The dot black line is the model temperature described in section 4.2. B) Rayleigh wave phase velocity changes predicted by the thermoelastic model discussed in section 4.2 at $6 \mathrm{~Hz}$ (black line) and $8 \mathrm{~Hz}$ (blue line) compared to the observed velocity variations. The two vertical red dashed lines are spaced of one martian year (668.6 Sols).

\section{DISCUSSION}

\subsection{Role of horizontal wavelength of temperature modulation}

When $l_{x}$ approaches the order of magnitude of the Rayleigh wavelength $\lambda_{r}$, the predictions of the thermo-elastic model differ significantly from the case $l_{x} \gg \lambda_{r}$. In the case $l_{x} \approx \lambda_{r}$, the transition from the first term (in $e^{-\gamma z}$ ) to the second term (in $e^{-k_{x} z}$ ), observed in Figure 8.C at about $4 \mathrm{~m}$ depth 
N. Compaire et al.

when $l_{x}=1000 \mathrm{~m}$, occurs much closer to the surface, where the amplitude of the velocity perturbations is still high (see $\mathrm{E}$ for the case $l_{x}=30 \mathrm{~m}$ ). Through the sensitivity to the shear wave speed, the $d v / v$ variations tend to be more influenced by the deeper areas, where the velocity perturbations are opposite in sign to those in the very near surface (see Figure A5.C and A5.D). More precisely, for sufficiently small $l_{x}$, the $d v / v$ variations induced by perturbations in $\mathrm{P}$ and $\mathrm{S}$ seismic velocities are of the same amplitude but are no longer in phase (we recall that the P-wavespeed kernel only sees the very near surface). As a result, the correlation between $d v / v$ and surface temperature is lost. From this analysis, it appears unlikely that $l_{x}$ is of the order of a few tens of meters or less. Such a conclusion needs of course confirmation from more realistic modeling of the local geological environment.

It is worth noting that the simple homogeneous half-space model employed so far is self-consistent in the sense that we do not impose the temperature at the surface but rather deduce it from the solar forcing and evaluate its impact in terms of thermal stresses and seismic velocities variations in the subsurface. In what follows, we will depart from complete self-consistency. First, we will impose the surface temperature forcing as deduced from local temperature measurements. Second, we will introduce a jump of seismic velocities at depth. The goal of this second assumption is to evaluate to what extent Rayleigh wave dispersion may help explain the frequency dependence of the amplitude of apparent velocity changes.

\subsection{Role of background seismic velocity profile}

In section 4, we showed that the thermo-elastic perturbation of the Martian regolith sensed by a wavefield composed of Rayleigh waves is a plausible explanation for the velocity variations observed at InSight. We now attempt at improving the model developed in the previous section to better account for the peculiarities of the observations.

First, we note that the surface temperature model given as an input to the thermoelastic model has an important impact on the shape of the output $\mathrm{dv} / \mathrm{v}$ curves. To better take into account the local conditions, we fitted empirically the characteristic pulsations, amplitudes and phases of the temperature forcing based on the so-called SCIT (Scientific Temperature of SEIS instrument, see C), as illustrated in Figure 10. Interestingly, we found that the best fit to the SCIT is provided by adopting a fundamental pulsation $\hat{\omega}=2 \pi \times 640^{-1}$ which is higher than the yearly pulsation. This suggests that transient meteorological phenomena may influence the ground temperature at the InSight landing site.

Second, the homogeneous half-space approximation for both the thermal and elastic properties is not realistic given the knowledge on the Martian subsurface structure at InSight. A better modeling of the velocity structure should likely incorporate a velocity contrast corresponding to the regolith to bedrock transition at 1 to 3 meters depth (Lognonn et al. 2020; Kenda et al. 2020; Onodera et al. 2021). 


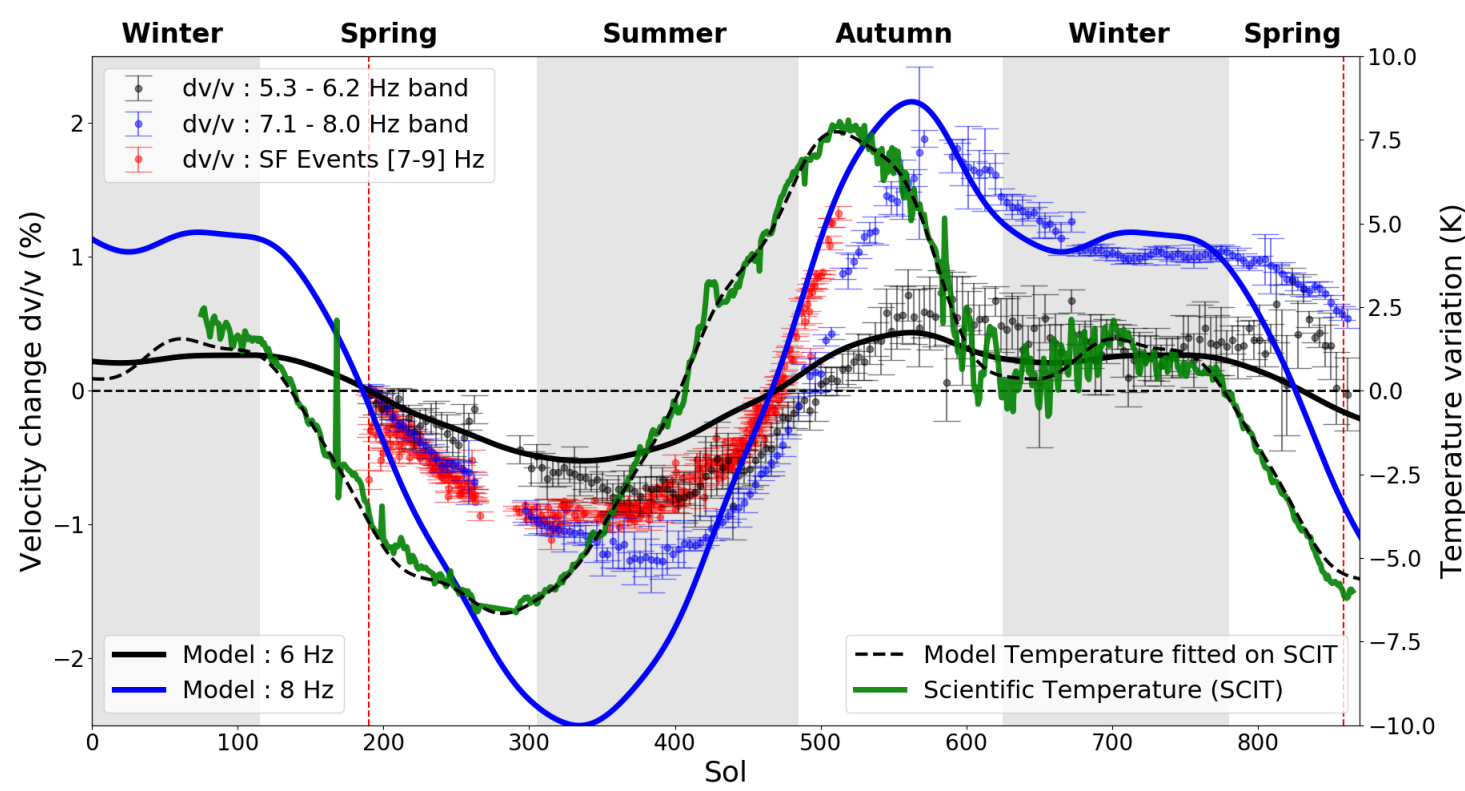

Figure 10. Rayleigh wave phase velocity changes predicted by the thermoelastic model considering a two-layer velocity profile at $6 \mathrm{~Hz}$ (black line) and $8 \mathrm{~Hz}$ (blue line) compared to the observed velocity variations. The green line is the daily average Scientific Temperature (see C). The black line is the model surface temperature based on a fit of the SCIT data and used as input for the thermoelastic model. The two vertical red dashed lines are spaced of one martian year (668.6 Sols).

A self-consistent thermoelastic model should also consider a change in the thermal properties between these two layers. Unfortunately, at the time of writing we do not have observational constraints on thermal parameters below $30 \mathrm{~cm}$ depth (Grott et al. 2021). Furthermore, a simple analytical solution to the thermo-elastic equations is not readily available in the literature for stratified media.

In order to test the effect of adding a layer at the top of the velocity model on the predicted Rayleigh wave phase velocity changes, we conduct a preliminary analysis with a non self-consistent thermoelastic structure. With uniform thermal properties, we consider a seismic S-wave velocity jump from $80 \mathrm{~m} / \mathrm{s}$ to $270 \mathrm{~m} / \mathrm{s}$ at 2.2 meters depth with a constant $v_{p} / v_{s}$ ratio in the two layers equal to 1.63 . While reasonable with respect to the subsurface models presented in Lognonn et al. (2020), these parameters have been determined empirically and should not be taken at face value. The results are shown in Figure 10 for $f=6 \mathrm{~Hz}$ (black solid line) and $f=8 \mathrm{~Hz}$ (blue solid line) for a surface input temperature based on a least-squares fit of the SCIT data. We notice that the frequency-dependent amplitude of the seismic velocity changes can be better modeled with a two-layer velocity profile. However, there remain discrepancies between the predicted and observed $d v / v$ around the velocity minimum (between Sol 200 and Sol 500). The model predictions are still ahead of the observations and the amplitude of the variation is too large for $d v / v$ at $8 \mathrm{~Hz}$. It should be kept in mind that the 
predictions shown in Figure 10 have been obtained with a non self-consistent model of thermoelastic properties. Also, as noted by Tsai (2011), thermoelastic models are two-dimensional and require a modulation of the surface temperature amplitude in the horizontal direction, that may be related to variations of thermal properties not taken into account in the model.

Note that the example shown in Figure 10 should not be interpreted as an attempt to constrain the subsurface velocity model but rather as a test of the effect of a layered medium on the apparent velocity change entailed by the thermoelastic response of the martian subsurface. What we illustrate is that the observations of relative velocity changes across a full martian year can provide strong constraints on the acousto-elastic properties $\left(\partial \rho v_{p}^{2} / \partial \sigma, \partial \rho v_{s}^{2} / \partial \sigma\right)$ as well as on the thermal and elastic structure of the martian regolith.

\subsection{Comparison with the Moon}

Temporal changes of velocity have previously been reported in the same seismic frequency band (6$8 \mathrm{~Hz}$ ) in the case of the Lunar regolith. Sens-Schnfelder \& Larose (2008) applied passive image interferometry to data acquired in the framework of the Lunar Seismic Profiling Experiment. They observed a clear anti-correlation between the surface temperature on the Moon and apparent velocity changes of the order of 0.1-0.2\% with a delay of approximately 14 days. Tanimoto et al. (2008) detected temporal variations in the group speed of Rayleigh waves retrieved by ambient noise cross-correlations. Their estimate of the amplitude and delay of the detected variations are broadly consistent with those reported by Sens-Schnfelder \& Larose (2008). To explain the anti-correlation between the temperature forcing and the seismic velocity response, Sens-Schnfelder \& Larose (2008) and Tanimoto et al. (2008) invoke the temperature dependence of elastic moduli (see e.g. Yang et al. (2019)). Because the amplitude of the seasonal temperature variations on Mars are approximately 10 times lower than the daily temperature variations on the Moon, the effect reported in Sens-Schnfelder \& Larose (2008) and Tanimoto et al. (2008) is expected to affect only marginally the seismic properties of the martian regolith (perturbations of the order of 0.01-0.02\%). The different responses of the Martian and Lunar regolith to thermal forcing remains to be understood. In the lunar case, we also expect to observe a thermo-elastic response due to the lateral variations of thermal properties and/or the thickness of the regolith. If so, the contrasting observations between Mars and Moon may be related to differences in the thermal and mechanical properties of the regoliths themselves, explainable by distinct formation processes (eolian deposit for Mars and crater ejecta for Moon). These are important topics for future investigations. 


\section{CONCLUSION}

Using a full martian year of SEIS data collected in the framework of the InSight mission on Mars, we report observations of seasonal variations in apparent seismic velocity perturbations from the analysis of the high-frequency seismic wavefield. The detected velocity changes are positively correlated with the surface temperature observed at InSight with a delay time of the order of 60 to 100 Sols. The magnitude of the velocity perturbation increases with the central frequency of the signals. Using a simple model of propagation based on Rayleigh waves in a half-space, we show that our observations are reasonably explained by a simple thermo-elastic model of the martian subsurface. In this model, the spatial and temporal modulations of the temperature at the surface induce time-dependent thermal elastic stresses at depth which modify the local seismic wavespeeds. These velocity perturbations can be directly observed in seismic event waveforms as well as in the spectra of high-frequency ambient vibrations. Our analysis suggests that the spatial modulation of the temperature compatible with the data should be of the order of $1000 \mathrm{~m}$ or more. In future works, the observations of velocity changes reported in this study could help constrain the seismic and thermoelastic properties of the subsurface under InSight up to about 20 meters depth through a joint inversion of the velocity and thermal conductivity profiles.

\section{ACKNOWLEDGMENTS}

This study is InSight contribution number 214. The authors acknowledge both "Universit Fdrale de Toulouse Midi Pyrnes" and the "Région Occitanie" for funding the PhD grant of Nicolas Compaire. The French authors acknowledge the French Space Agency CNES and ANR (ANR-14-CE36-001202 and ANR-19-CE31-0008-08) for funding the InSight Science analysis. We acknowledge NASA, CNES, their partner agencies and Institutions (UKSA, SSO, DLR, JPL, IPGP-CNRS, ETHZ, IC, MPSMPG) and the flight operations team at JPL, SISMOC, MSDS, IRIS-DMC and PDS for providing SEED SEIS data. The design, building of and research into the HP3 has been supported by the German Aerospace Center DLR, NASA, the Austrian Academy of Science AW, and the Polish Academy of Science PAN.

SEIS data are referenced at http://dx.doi.org/10.18715/SEIS.INSIGHT.XB_2016. The Mars Quake service (MQS) catalogue of events used in this contribution is the Mars Seismic Catalogue, InSight Mission, V6 acknowledging ETHZ, IPGP, JPL, ICL, ISAE-Supaero, MPS, and the University of Bristol. It is available at http://doi.org/10.12686/a11.

The Mars data of the HP3 radiometer (RAD) are archived on the Planetary Data System. It is available at https://pds-geosciences.wustl.edu/missions/insight/hp3rad.htm 


\section{REFERENCES}

Aki, K. \& Richards, P. G., 2002. Quantitative seismology.

Akkermans, E. \& Montambaux, G., 2007. Mesoscopic physics of electrons and photons, Cambridge university press.

Banerdt, W. B., Smrekar, S. E., Banfield, D., Giardini, D., Golombek, M., Johnson, C. L., Lognonné, P., Spiga, A., Spohn, T., Perrin, C., Stähler, S. C., Antonangeli, D., Asmar, S., Beghein, C., Bowles, N., Bozdag, E., Chi, P., Christensen, U., Clinton, J., Collins, G. S., Daubar, I., Dehant, V., Drilleau, M., Fillingim, M., Folkner, W., Garcia, R. F., Garvin, J., Grant, J., Grott, M., Grygorczuk, J., Hudson, T., Irving, J. C. E., Kargl, G., Kawamura, T., Kedar, S., King, S., Knapmeyer-Endrun, B., Knapmeyer, M., Lemmon, M., Lorenz, R., Maki, J. N., Margerin, L., McLennan, S. M., Michaut, C., Mimoun, D., Mittelholz, A., Mocquet, A., Morgan, P., Mueller, N. T., Murdoch, N., Nagihara, S., Newman, C., Nimmo, F., Panning, M., Pike, W. T., Plesa, A.-C. Rodriguez, S., Rodriguez-Manfredi, J. A., Russell, C. T., Schmerr, N., Siegler, M., Stanley, S., Stutzmann, E., Teanby, N., Tromp, J., van Driel, M., Warner, N., Weber, R., \& Wieczorek, M., 2020. Initial results/from the InSight mission on mars, Nature Geoscience, 13(3), 183-189.

Berger, J., 1975. A note on thermoelastic strains and tilts, Journal of Geophysical Research, 80(2), 274-277. Bottelin, P., Baillet, L., Larose, E., Jongmans, D., Hantz, D., Brenguier, O., Cadet, H., \& Helmstetter, A., 2017. Monitoring rock reinforcement works with ambient vibrations: La bourne case study (vercors, france), Engineering Geology, 226, 136-145.

Ceylan, S., Clinton, J. F., Giardini, D., Böse, M., Charalambous, C., van Driel, M., Horleston, A., Kawamura, T., Khan, A., Orhand-Mainsant, G., Scholz, J.-R., Stähler, S. C., Euchner, F., Banerdt, W. B., Lognonné, P., Banfield, D., Beucler, E., Garcia, R. F., Kedar, S., Panning, M. P, Pike, W. T., Smrekar, S. E., Spiga, A., Dahmen, N. L., Hurst, K., Stott, A. E., Lorenz, R. D., Schimmel, M., Stutzmann, E., ten Pierick, J., Conejero, V., Pardo, C., \& Perrin, C., 2021. Companion guide to the marsquake catalog from InSight, sols 0-478: Data content and non-seismic events, Physics of the Earth and Planetary Interiors, 310, 106597.

Clarke, D., Zaccarelli, L., Shapiro, N. M., \& Brenguier, F., 2011. Assessment of resolution and accuracy of the Moving Window Cross Spectral technique for monitoring crustal temporal variations using ambient seismic noise: MWCS: assessment of resolution and accuracy, Geophysical Journal International, 186(2), 867-882. Compaire, N., Margerin, L., Garcia, R.,Pinot, B., Calvet, M., Orhand-Mainsant, G., Kim, D., Lekic, V., Tauzin, B., Schimmel, M., Stutzmann, E., Knapmeyer-Endrun, B., Lognonné, P., Pike, W. T., Schmerr, N., Gizon, L., \& Banerdt, W. B., 2021. Autocorrelation of the ground vibrations recorded by the SEIS-InSight seismometer on mars., Journal of Geophysical Research: Planets, p. e2020JE006498.

Dahmen, N. L., Clinton, J. F., Ceylan, S., van Driel, M., Giardini, D., Khan, A., Stähler, S. C., Böse, M., Charalambous, C., Horleston, A., Kawamura, T., Orhand-Mainsant, G., Scholz, J.-R., Euchner, F., Pike, W. T., Weber, R. C., Lognonné, P., \& Banerdt, W. B., 2021a. Super high frequency events: A new class of events recorded by the InSight seismometers on mars, Journal of Geophysical Research: Planets, 126(2),

e2020JE006599.

Dahmen, N. L., Zenhäusern, G., Clinton, J. F., Giardini, D., Stähler, S. C., Ceylan, S., Charalambous, C., van 
Driel, M., Hurst, K. J., Kedar, S., Lognonné, P., Murdoch, N., Myhill, R., Panning, M. P., Pike, W. T., Schimmel, M., Schmelzbach, C., Scholz, J.-R., Stott, A. E., Stutzmann, E., \& Banerdt, W. B., 2021b. Resonances and lander modes observed by InSight on mars (1-9 hz), Bulletin of the Seismological Society of America.

Dainty, A. M. \& Toksz, M., 1981. Seismic codas on the earth and the moon: a comparison, Physics of the Earth and Planetary Interiors, 26(4), 250-260.

De Plaen, R. S. M., Lecocq, T., Caudron, C., Ferrazzini, V., \& Francis, O., 2016. Single-station monitoring of volcanoes using seismic ambient noise, Geophysical Research Letters, 43(16), 8511-8518.

Deng, S. \& Levander, A., 2020. Autocorrelation reflectivity of mars, Geophysical Research Letters, 47(16), e2020GL089630.

Derode, A., Tourin, A., \& Fink, M., 2001. Random multiple scattering of ultrasound. ii. is time reversal a self-averaging process?, Physical Review E, 64(3), 036606.

Driel, M., Ceylan, S., Clinton, J. F., Giardini, D., Horleston, A., Margerin, L., Stähler, S. C., Böse, M., Charalambous, C., Kawamura, T., Khan, A., Orhand-Mainsant, G., Scholz, J.-R., Euchner, F., Knapmeyer,M., Schmerr, N., Pike, W. T., Lognonné, P., \& Banerdt, W. B., 2021. High-frequency seismic events on mars observed by InSight, Journal of Geophysical Research: Planets, 126(2).

Giardini, D., Lognonn, P., Banerdt, W. B., Pike, W. T., Christensen, U., Ceylan, S., Clinton, J. F., van Driel, M., Sthler, S. C., Bse, M., Garcia, R. F., Khan, A., Panning, M., Perrin, C., Banfield, D., Beucler, E., Charalambous, C., Euchner, F., Horleston, A., Jacob, A., Kawamura, T., Kedar, S., Maínsant, G., Scholz, J.-R., Smrekar, S. E., Spiga, A., Agard, C., Antonangeli, D., Barkaoui, S., Barrett, E., Combes, P., Conejero, V., Daubar, I., Drilleau, M., Ferrier, C., Gabsi, T., Gudkova, T., Hurst, K., Karakostas, F., King, S., Knapmeyer, M., Knapmeyer-Endrun, B., Llorca-Cejudo, R., Lucas, A., Luno, L., Margerin, L., McClean, J. B., Mimoun, D., Murdoch, N., Nimmo, F., Nonon, M., Pardo, C., Rivoldini, A., Manfredi, J. A. R., Samuel, H., Schimmel, M., Stott, A. E., Stutzmann, E., Teanby, N., Warren, T., Weber, R. C., Wieczorek, M., \& Yana, C., 2020. The seismicity of Mars, Nature Geoscience, 13(3), 205-212.

Grott, M., Spohn, T., Knollenberg, J., Krause, C., Hudson, T. L., Piqueux, S., Müller, N., Golombek, M., Vrettos, C., Marteau, E., Nagihara, S., Morgan, P., Murphy, J. P., Siegler, M., King, S. D., Smrekar, S. E., \& Banerdt, W. B., 2021. Thermal conductivity of the martian soil at the InSight landing site from HP 3 active heating experiments, Journal of Geophysical Research: Planets, 126(7).

Hadziioannou, C., Larose, E., Coutant, O., Roux, P., \& Campillo, M., 2009. Stability of monitoring weak changes in multiply scattering media with ambient noise correlation: Laboratory experiments, The Journal of the Acoustical Society of America, 125(6), 3688-3695.

Hobiger, M., Wegler, U., Shiomi, K., \& Nakahara, H., 2014. Single-station cross-correlation analysis of ambient seismic noise: application to stations in the surroundings of the 2008 iwate-miyagi nairiku earthquake, Geophysical Journal International, 198(1), 90-109.

InSight Mars SEIS Data Service, 2019. Seis raw data, insight mission.

InSight Marsquake Service, 2021. Mars seismic catalogue, insight mission; v6 2021-04-01 [data set].

Kenda, B., Drilleau, M., Garcia, R. F., Kawamura, T., Murdoch, N., Compaire, N., Lognonné, P., Spiga, A., 
Widmer-Schnidrig, R., Delage, P., Ansan, V., Vrettos, C., Rodriguez, S., Banerdt, W. B., Banfield, D., Antonangeli, D., Christensen, U., Mimoun, D., Mocquet, A., \& Spohn, T., 2020. Subsurface structure at the InSight landing site from compliance measurements by seismic and meteorological experiments, Journal of Geophysical Research: Planets, 125(6).

Knapmeyer-Endrun, B., Panning, M. P., Bissig, F., Joshi, R., Khan, A., Kim, D., Lekić, V., Tauzin, B., Tharimena, S., Plasman, M., Compaire, N., Garcia, R. F., Margerin, L., Schimmel, M., Stutzmann, É., Schmerr, N., Bozdă̆, E., Plesa, A.-C., Wieczorek, M. A., Broquet, A., Antonangeli, D., McLennan, S. M., Samuel, H., Michaut, C., Pan, L., Smrekar, S. E., Johnson, C. L., Brinkman, N., Mittelholz, A., Rivoldini, A., Davis, P. M., Lognonné, P., Pinot, B., Scholz, J.-R., Stähler, S., Knapmeyer, M., van Driel, M., Giardini, D., \& Banerdt, W. B., 2021. Thickness and structure of the martian crust from InSight seismic data, Science, 373(6553), 438-443.

Kopp, E., Müller, N., Grott, M., Walter, I., Knollenberg, J., Hänschke, F., Kessler, E., \& Meyer, H.-G., 2016. HP3-RAD: a compact radiometer design with on-site calibration for in-situ exploration, in Infrared Remote Sensing and Instrumentation XXIV, SPIE.

Larose, E., Khan, A., Nakamura, Y., \& Campillo, M., 2005. Lunar subsurface investigated from correlation of seismic noise, Geophysical Research Letters, 32(16), n/a.

Larose, E., Roux, P., Campillo, M., \& Derode, A., 2008. Fluctuations of correlations and green's function reconstruction: Role of scattering, Journal of Applied Physics, 103(11), 114907.

Larose, E., Planes, T., Rossetto, V., \& Margerin, L., 2010. Locating a small change in a multiple scattering environment, Applied Physics Letters, 96(20), 204101.

Liu, Z., Huang, J., \& Li, J., 2010. Comparison of four techniques for estimating temporal change of seismic velocity with passive image interferometry, Earthquake Science, 23(5), 511-518.

Lobkis, O. I. \& Weaver, R. L., 2001. On the emergence of the Greens function in the correlations of a diffuse field, The Journal of the Acoustical Society of America, 110(6), 3011-3017.

Lognonn, P., Banerdt, W. B., Giardini, D., Píke, W. T., Christensen, U., Laudet, P., de Raucourt, S., Zweifel, P., Calcutt, S., Bierwirth, M., Hurst, K. J., Ijpelaan, F., Umland, J. W., Llorca-Cejudo, R., Larson, S. A., Garcia, R. F., Kedar, S., Knapmeyer-Endrun, B., Mimoun, D., Mocquet, A., Panning, M. P., Weber, R. C., Sylvestre-Baron, A., Pont, G., Verdier, N., Kerjean, L., Facto, L. J., Gharakanian, V., Feldman, J. E., Hoffman, T. L., Klein, D. B., Klein, K., Onufer, N. P., Paredes-Garcia, J., Petkov, M. P., Willis, J. R., Smrekar, S. E., Drilleau, M., Gabsi, T., Nebut, T., Robert, O., Tillier, S., Moreau, C., Parise, M., Aveni, G., Ben Charef, S., Bennour, Y., Camus, T., Dandonneau, P. A., Desfoux, C., Lecomte, B., Pot, O., Revuz, P., Mance, D., tenPierick, J., Bowles, N. E., Charalambous, C., Delahunty, A. K., Hurley, J., Irshad, R., Liu, H., Mukherjee, A. G., Standley, I. M., Stott, A. E., Temple, J., Warren, T., Eberhardt, M., Kramer, A., Khne, W., Miettinen, E.PP., Monecke, M., Aicardi, C., Andr, M., Baroukh, J., Borrien, A., Bouisset, A., Boutte, P., Brethom, K., Brysbaert, C., Carlier, T., Deleuze, M., Desmarres, J. M., Dilhan, D., Doucet, C., Faye, D., Faye-Refalo, N., Gonzalez, R., Imbert, C., Larigauderie, C., Locatelli, E., Luno, L., Meyer, J.-R., Mialhe, F., Mouret, J. M., Nonon, M., Pahn, Y., Paillet, A., Pasquier, P., Perez, G., Perez, R., Perrin, L., Pouilloux, B., Rosak, 
A., Savin deLarclause, I., Sicre, J., Sodki, M., Toulemont, N., Vella, B., Yana, C., Alibay, F., Avalos, O. M., Balzer, M. A., Bhandari, P., Blanco, E., Bone, B. D., Bousman, J. C., Bruneau, P., Calef, F. J., Calvet, R. J., DAgostino, S. A., delos Santos, G., Deen, R. G., Denise, R. W., Ervin, J., Ferraro, N. W., Gengl, H. E., Grinblat, F., Hernandez, D., Hetzel, M., Johnson, M. E., Khachikyan, L., Lin, J. Y., Madzunkov, S. M., Marshall, S. L., Mikellides, I. G., Miller, E. A., Raff, W., Singer, J. E., Sunday, C. M., Villalvazo, J. F., Wallace, M. C., Banfield, D., Rodriguez-Manfredi, J. A., Russell, C. T., Trebi-Ollennu, A., Maki, J. N., Beucler, E., Bse, M., Bonjour, C., Berenguer, J. L., Ceylan, S., Clinton, J., Conejero, V., Daubar, I., Dehant, V., Delage, P., Euchner, F., Estve, I., Fayon, L., Ferraioli, L., Johnson, C. L., Gagnepain-Beyneix, J., Golombek, M., Khan, A., Kawamura, T., Kenda, B., Labrot, P., Murdoch, N., Pardo, C., Perrin, C., Pou, L., Sauron, A., Savoie, D., Sthler, S., Stutzmann, E., Teanby, N. A., Tromp, J., van Driel, M., Wieczorek, M., WidmerSchnidrig, R., \& Wookey, J., 2019. SEIS: Insights Seismic Experiment for Internal Structure of Mars, Space Science Reviews, 215(1), 170.

Lognonn, P., Banerdt, W. B., Pike, W. T., Giardini, D., Christensen, U., Garcia, R. F., Kawamura, T., Kedar, S., Knapmeyer-Endrun, B., Margerin, L., Nimmo, F., Panning, M., Tauzin, B., Scholz, J.-R., Antonangeli, D., Barkaoui, S., Beucler, E., Bissig, F., Brinkman, N., Calvet, M., Ceylan, S., Charalambous, C., Davis, P., van Driel, M., Drilleau, M., Fayon, L., Joshi, R., Kenda, B., Khan, A., Knapmeyer, M., Lekic, V., McClean, J., Mimoun, D., Murdoch, N., Pan, L., Perrin, C., Pinot, B., Pou, L., Menina, S., Rodriguez, S., Schmelzbach, C., Schmerr, N., Sollberger, D., Spiga, A., Sthler, S., Stott, A., Stutzmann, E., Tharimena, S., Widmer-Schnidrig, R., Andersson, F., Ansan, V., Beghein, C., Bse, M., Bozdag, E., Clinton, J., Daubar, I., Delage, P., Fuji, N., Golombek, M., Grott, M., Horleston, A., Hurst, K., Irving, J., Jacob, A., Knollenberg, J., Krasner, S., Krause, C., Lorenz, R., Michaut, C., Myhill, R., Nissen-Meyer, T., ten Pierick, J., Plesa, A.-C., QuantinNataf, C., Robertsson, J., Rochas, L., Schimmel, M., Smrekar, S., Spohn, T., Teanby, N., Tromp, J., Vallade, J., Verdier, N., Vrettos, C., Weber, R., Banfield, D., Barrett, E., Bierwirth, M., Calcutt, S., Compaire, N., Johnson, C., Mance, D., Euchner, F., Kerjean, L., Mainsant, G., Mocquet, A., Rodriguez Manfredi, J. A., Pont, G., Laudet, P., Nebut, T., de Raucourt, S., Robert, O., Russell, C. T., Sylvestre-Baron, A., Tillier, S., Warren, T., Wieczorek, M., Yana, C., \& Zweifel, P., 2020. Constraints on the shallow elastic and anelastic structure of Mars from InSight seismic data, Nature Geoscience, 13(3), 213-220.

Menina, S., Margerin, L., Kawamura, T., Lognonné, P., Marti, J., Drilleau, M., Calvet, M., Compaire, N., Garcia, R., Karakostas, F., Schmerr, N., van Driel, M., Stähler, S. C., Plasman, M., Giardini, D., Carrasco, S., Knapmeyer-Endrun, B., Sainton, G., \& Banerdt, W. B., 2021. Energy envelope and attenuation characteristics of high-frequency (HF) and very-high-frequency (VF) martian events, Bulletin of the Seismological Society of America, 111(6), 3016-3034.

Moreau, L., Stehly, L., Boué, P., Lu, Y., Larose, E., \& Campillo, M., 2017. Improving ambient noise correlation functions with an SVD-based wiener filter, Geophysical Journal International, 211(1), 418-426.

Mueller, N., Knollenberg, J., Grott, M., Kopp, E., Walter, I., Krause, C., Hudson, T., Spohn, T., \& Smrekar, S., 2020. Calibration of the HP3 radiometer on InSight, Earth and Space Science, 7(5).

Mueller, N., Piqueux, S., Lemmon, M., Maki, J., Lorenz, R. D., Grott, M., Spohn, T., Smrekar, S. E., Knol- 
lenberg, J., Hudson, T. L., Krause, C., Millour, E., Forget, F., Golombek, M., Hagermann, A., Attree, N., Siegler, M., \& Banerdt, W. B., 2021. Near surface properties of martian regolith derived from insight hp3-rad temperature observations during phobos transits, Geophysical Research Letters, 48(15), e2021GL093542.

Onodera, K., Kawamura, T., Lognonné, P., Drilleau, M., Murdoch, N., Plasman, M., Perrin, C., Schimmel, M., Stutzmann, E., Xu, Z., Garcia, R., Vincent, M., Jacob, A., Lucas, A., \& Rodriguez, S., 2021. Subsurface structure around the insight landing site: Compliance analysis for large convective vortices observed during one martian year operation, Bulletin of the Seismological Society of America, p. na.

Oren, C. \& Nowack, R. L., 2017. Seismic body-wave interferometry using noise autocorrelations for crustal structure, Geophysical Journal International, 208(1), 321-332.

Pacheco, C. \& Snieder, R., 2005. Time-lapse travel time change of multiply scattered acoustic waves, The Journal of the Acoustical Society of America, 118(3), 1300-1310.

Panning, M. P., Pike, W. T., Lognonné, P., Banerdt, W. B., Murdoch, N., Banfield, D., Charalambous, C., Kedar, S., Lorenz, R. D., Marusiak, A. G., McClean, J. B., Nunn, C., Stähler, S. C., Stott, A. E., \& Warren, T., 2020. On-deck seismology: Lessons from InSight for future planetary seismology, Journal of Geophysical Research: Planets, 125(4).

Piqueux, S., Mller, N., Grott, M., Siegler, M., Millour, E., Forget, F., Lemmon, M., Golombek, M., Williams, N., Grant, J., Warner, N., Ansan, V., Daubar, I., Knollenberg, J., Maki, J., Spiga, A., Banfield, D., Spohn, T., Smrekar, S., \& Banerdt, B., 2021. Soil thermophysical properties near the insight lander derived from 50 sols of radiometer measurements, Journal of Geophysical Research: Planets, 126(8), e2021JE006859.

Planès, T., Larose, E., Margerin, L., Rossetto, V., \& Sens-Schoenfelder, C., 2014. Decorrelation and phaseshift of coda waves induced by local changes: multiple scattering approach and numerical validation, Waves in Random and Complex Media, 24(2), 99-125.

Pou, L., Mimoun, D., Lognonne, P., Garcia, R. F., Karatekin, O., Nonon-Latapie, M., \& Llorca-Cejudo, R., 2018. High precision SEIS calibration for the InSight míssion and its applications, Space Science Reviews, 215(1).

Poupinet, G., Ellsworth, W. L., \& Frechet, J. 1984. Monitoring velocity variations in the crust using earthquake doublets: An application to the calaveras fault, california, Journal of Geophysical Research: Solid Earth, 89, 5719-5731.

Poupinet, G., Got, J.-L., \& Brenguier, F., 2008. Monitoring temporal variations of physical properties in the crust by cross-correlating the waveforms of seismic doublets, Advances in geophysics, 50, 373-399.

Richter, T., Sens-Schnfelder, C., Kind, R., \& Asch, G., 2014. Comprehensive observation and modeling of earthquake and temperature-related seismic velocity changes in northern chile with passive image interferometry, Journal of Geophysical Research: Solid Earth, 119(6), 4747-4765.

Schimmel, M., Stutzmann, E., Lognonné, P., Compaire, N., Davis, P., Drilleau, M., Garcia, R., Kim, D., Knapmeyer-Endrun, B., Lekic, V., Margerin, L., Panning, M., Schmerr, N., Scholz, J. R., Spiga, A., Tauzin, B., \& Banerdt, B., 2021. Seismic noise autocorrelations on mars, Earth and Space Science.

Scholz, J.-R., Widmer-Schnidrig, R., Davis, P., Lognonné, P., Pinot, B., Garcia, R. F., Hurst, K., Pou, L., 
Nimmo, F., Barkaoui, S., de Raucourt, S., Knapmeyer-Endrun, B., Knapmeyer, M., Orhand-Mainsant, G., Compaire, N., Cuvier, A., Beucler, É., Bonnin, M., Joshi, R., Sainton, G., Stutzmann, E., Schimmel, M., Horleston, A., Böse, M., Ceylan, S., Clinton, J., van Driel, M., Kawamura, T., Khan, A., Stähler, S. C., Giardini, D., Charalambous, C., Stott, A. E., Pike, W. T., Christensen, U. R., \& Banerdt, W. B., 2020. Detection, analysis, and removal of glitches from InSights seismic data from mars, Earth and Space Science, 7(11).

Sens-Schnfelder, C. \& Larose, E., 2008. Temporal changes in the lunar soil from correlation of diffuse vibrations, Physical Review E, 78(4).

Sens-Schönfelder, C. \& Eulenfeld, T., 2019. Probing the in situ elastic nonlinearity of rocks with earth tides and seismic noise, Phys. Rev. Lett., 122, 138501.

Sens-Schönfelder, C. \& Wegler, U., 2006. Passive image interferometry and seasonal variations of seismic velocities at merapi volcano, indonesia, Geophysical Research Letters, 33(21).

Shapiro, B., 1986. Large intensity fluctuations for wave propagation in random media, Physical review letters, 57(17), 2168.

Shapiro, N. M., 2005. High-resolution surface-wave tomography from ambient seismic noise, Science, 307(5715), 1615-1618.

Siegler, M. A., Smrekar, S. E., Grott, M., Piqueux, S., Mueller, N., Williams, J.-P., Plesa, A.-C., \& Spohn, T., 2017. The InSight mars lander and its effect on the subsurface thermal environment, Space Science Reviews, 211(1-4), 259-275.

Snieder, R., Grêt, A., Douma, H., \& Scales, J., 2002. Coda wave interferométry for estimating nonlinear behavior in seismic velocity, Science, 295(5563), 2253-2255.

Stutzmann, E., Schimmel, M., Lognonné, P., Horleston, A., Ceylan, S., van Driel, M., Stahler, S., Banerdt, B., Calvet, M., Charalambous, C., Clinton, J., Drilleau, M., Fayon, L., Garcia, R. F., Giardini, D., Hurst, K., Jacob, A., Kawamura, T., Kenda, B., Margerin, L., Murdoch, N., Panning, M., Pike, T., Scholz, J.-R., $\&$ Spiga, A., 2021. The polarization of ambient noise on mars, Journal of Geophysical Research: Planets, 126(1).

Suemoto, Y., Ikeda, T., \& Tsuji, T., 2020. Temporal variation and frequency dependence of seismic ambient noise on mars from polarization analysis, Geophysical Research Letters, 47(13).

Tanimoto, T., Eitzel, M., \& Yano, T, 2008. The noise cross-correlation approach for apollo 17 LSPE data: Diurnal change in seismic parameters in shallow lunar crust, Journal of Geophysical Research, 113(E8).

Tsai, V. C., 2011. A módel for seasonal changes in GPS positions and seismic wave speeds due to thermoelastic and hydrologic variations, Journal of Geophysical Research: Solid Earth, 116.

van Driel, M., Ceylan, S., Clinton, J. F., Giardini, D., Horleston, A., Margerin, L., Stähler, S. C., Böse, M., Charalambous, C., Kawamura, T., et al., 2021. High-frequency seismic events on mars observed by insight, Journal of Geophysical Research: Planets, 126(2), e2020JE006670.

Weaver, R. L. \& Lobkis, O. I., 2004. Diffuse fields in open systems and the emergence of the greens function (1), The Journal of the Acoustical Society of America, 116(5), 2731-2734.

Winkler, K. W. \& McGowan, L., 2004. Nonlinear acoustoelastic constants of dry and saturated rocks, Journal 
of Geophysical Research: Solid Earth, 109(B10).

Yang, J., Fu, L.-Y., Fu, B.-Y., Wang, Z., \& Hou, W., 2019. On the nonlinear temperature dependence of elastic constants and wave velocities for solid media with applications to geologic materials, The Journal of the Acoustical Society of America, 146(3), 1556-1567.

Zhan, Z., Tsai, V. C., \& Clayton, R. W., 2013. Spurious velocity changes caused by temporal variations in ambient noise frequency content, Geophysical Journal International, 194(3), 1574-1581. 


\section{APPENDIX A: SF EVENTS FAMILIES}

The list of the SF events present in each family discussed in section 2.1 is given in Table A1. The event names follow the MarsQuake Service (MQS) nomenclature (InSight Marsquake Service 2021). The SF events were detected late compared to other types of events. To distinguish them in the MQS catalog they have been assigned the letter "T". The number in the middle of the name corresponds to the number of the Sol. The lowercase letter is used to distinguish events that took place on the same Sol.

\section{A1 Frequency content and signal-to-noise ratio (SNR)}

The accuracy of the Moving-Window Cross-Spectral technique (MWCS) used in section 2.2 depends on the Signal-to-Noise Ratio (SNR) of the seismic events (Clarke et al. 2011). We thus conduct a SNR analysis on the SF events in each family to determine the best component and best frequency band to perform the monitoring of the relative traveltime change of the seismic waves.

The results of this analysis are shown in Figure A1 for families $\mathrm{C} 0$ and D0 and in Figure A2 for the others families. For each event the signal window is the 50 secondes time-window given by the MQS catalogue (InSight Marsquake Service 2021). The noise window is the 50 secondes time-window just before the start-time of the signal window. The Amplitude Spectral Density (ASD) are computed using a Welch algorithm with 400 samples per segment (20 secondes) and 50\% of overlap. The SNR is the ratio of the ASD in the signal window and the ASD in the noise window smoothed with a smoothing parameter of $1 \mathrm{~Hz}$.

It result from this analysis that the quality D families have their higher SNR on the East component between $7 \mathrm{~Hz}$ and $9 \mathrm{~Hz}$. We see in Figure A1 and A2 that quality $\mathrm{C}$ families have also high SNR between $5 \mathrm{~Hz}$ and $7 \mathrm{~Hz}$ but in order to compare consistent observations we choose to analyse all the events using the same configuration. 


\section{N. Compaire et al.}

Table A1. List of SF events in each family. Event names referring to the catalog V6 by the MQS (InSight Marsquake Service 2021)

SF families

\begin{tabular}{|c|c|c|c|c|c|c|c|c|c|c|}
\hline $\mathrm{C} 0$ & & $\mathrm{C} 1$ & $\mathrm{C} 2$ & D0 & & D1 & D2 & D3 & & D4 \\
\hline T0190a & $\mathrm{T} 0234 \mathrm{c}$ & T0345a & T0488a & T0190b & T0261b & $\mathrm{T} 0355 \mathrm{~b}$ & T0375a & T0416f & T0450d & $\mathrm{T} 0454 \mathrm{c}$ \\
\hline T0192a & $\mathrm{T} 0235 \mathrm{a}$ & T0347a & T0491a & T0191a & $\mathrm{T} 0262 \mathrm{c}$ & T0361d & T0376b & T0419a & T0452b & T0455a \\
\hline T0193a & T0236a & T0349a & T0492a & T0192b & T0263b & $\mathrm{T} 0362 \mathrm{c}$ & T0378b & $\mathrm{T} 0422 \mathrm{c}$ & T0453a & T0456a \\
\hline T0196а & T0237a & T0350a & T0493a & T0195b & T0266b & $\mathrm{T} 0365 \mathrm{c}$ & T0382e & $\mathrm{T} 0427 \mathrm{c}$ & T0453d & T0457a \\
\hline T0197b & T0238a & T0351b & T0494a & T0198c & T0291a & T0366a & T0383b & T0428e & T0454a & T0458b \\
\hline T0198b & $\mathrm{T} 0239 \mathrm{~b}$ & T0353a & T0495a & T0199b & Т0293a & $\mathrm{T} 0367 \mathrm{~b}$ & T0385d & T0429b & $\mathrm{T} 0454 \mathrm{~d}$ & T0459b \\
\hline T0199a & $\mathrm{T} 0240 \mathrm{~b}$ & T0355a & T0496a & T0200c & T0297a & $\mathrm{T} 0368 \mathrm{~d}$ & T0390d & $\mathrm{T} 0429 \mathrm{~d}$ & T0456b & T0460b \\
\hline T0200b & $\mathrm{T} 0241 \mathrm{~b}$ & T0356a & T0497b & T0201b & T0298a & $\mathrm{T} 0369 \mathrm{~d}$ & T0391b & T0430d & T0456c & T0461d \\
\hline T0201a & T0242b & T0358a & T0498a & $\mathrm{T} 0202 \mathrm{~b}$ & T0300a & T0369e & T0394c & T0430e & T0457b & T0462b \\
\hline T0202a & T0243b & T0359a & T0500b & T0204a & $\mathrm{T} 0302 \mathrm{a}$ & $\mathrm{T} 0371 \mathrm{~b}$ & T0398d & T0431e & $\mathrm{T} 0458 \mathrm{c}$ & T0463a \\
\hline T0203a & T0244a & T0360a & T0501a & T0205b & T0303a & T0372c & T0399c & T0433d & T0458d & T046 \\
\hline T0205a & $\mathrm{T} 0245 \mathrm{c}$ & $\mathrm{T} 0361 \mathrm{c}$ & T0505a & T0206b & T0305a & $\mathrm{T} 0374 \mathrm{~b}$ & T0400a & T0433e & $461 \mathrm{f}$ & \\
\hline T0206a & T0246a & T0362b & T0506a & T0209b & T0306a & T0378a & T0401b & $\mathrm{T} 0433 \mathrm{~g}$ & & \\
\hline T0207a & T0247a & T0363a & T0507a & T0210b & Т0308a & $\mathrm{T} 0380 \mathrm{~b}$ & $\mathrm{~T} 0402 \mathrm{c}$ & T0434b & & \\
\hline T0208a & Т0249c & T0364a & T0508a & $\mathrm{T} 0211 \mathrm{c}$ & T0309b & T0381c & T0403f & T0435f & & T0468a \\
\hline T0209a & T0250a & T0365a & T0512a & T0213b & T0310a & T0382d & T0404f & & & T0468c \\
\hline T0210a & T0251a & T0368a & & T0214b & T0313a & T0383a & T0405d & & & T0469b \\
\hline T0211b & T0252a & T0368b & & $\mathrm{T} 0215 \mathrm{~b}$ & T0314a & $\mathrm{T} 0384 \mathrm{~d}$ & $\mathrm{~T} 0407 \mathrm{~h}$ & $\mathrm{~T}$ & & T0470d \\
\hline T0212a & $\mathrm{T} 0253 \mathrm{~b}$ & T0369a & & $\mathrm{T} 0218 \mathrm{~b}$ & $\mathrm{~T} 0315 \mathrm{a}$ & T0385c & & T0438c & & T0471a \\
\hline T0213a & T0254a & T0370a & & T0219b & T0316a & & T0410b & T0438e & & T0472a \\
\hline T0214a & $\mathrm{T} 0255 \mathrm{a}$ & T0372a & & T0220b & T0317a & & $\mathrm{T} 0413 \mathrm{e}$ & Т0439c & & $\mathrm{T} 0473 \mathrm{c}$ \\
\hline T0215a & Т0256а & T0390a & & $\mathrm{T} 0223 \mathrm{c}$ & T03 & & $\mathrm{T} 0414 \mathrm{c}$ & T0440b & & T0473d \\
\hline T0216a & T0261a & & & $\mathrm{T} 0225 \mathrm{c}$ & & T0394d & T0415a & T0440d & & T0474a \\
\hline T0217a & T0262a & & & $231 \mathrm{~b}$ & TO & T0396b & T0416e & T0441a & & T0475a \\
\hline T0218a & T0263a & & & & T0321a & T0398c & $\mathrm{T} 0417 \mathrm{c}$ & T0442a & & T0475b \\
\hline T0219a & & & & & T0322a & $\mathrm{T} 0400 \mathrm{c}$ & T0419c & $\mathrm{T} 0442 \mathrm{f}$ & & T0476b \\
\hline T0220a & & & & & T0323a & $\mathrm{T} 0401 \mathrm{c}$ & T0420c & Т0443a & & T0478b \\
\hline T0221a & & & & T0239c & T0324a & $\mathrm{T} 0405 \mathrm{e}$ & T0421b & T0443d & & T0479c \\
\hline T0222a & & & & T0240d & T0325a & & T0424a & T0444a & & T0480f \\
\hline T0223b & & & & $\mathrm{T} 0241 \mathrm{c}$ & T0326a & & & T0444b & & T0481b \\
\hline T0224c & & & & $\mathrm{T} 0242 \mathrm{c}$ & T0328a & & & T0444d & & T0482c \\
\hline $\mathrm{T} 0225 \mathrm{a}$ & & & & T0244b & T0331b & & & T0445a & & T0483b \\
\hline & & & & T0245b & T0333a & & & T0446b & & T0487b \\
\hline & & & & $\mathrm{T} 0250 \mathrm{~b}$ & T0334a & & & T0446d & & T0489a \\
\hline 28 & & & & $\mathrm{~T} 0252 \mathrm{~b}$ & T0337a & & & $\mathrm{T} 0447 \mathrm{~b}$ & & T0492b \\
\hline Т0229a & & & & T0253a & $\mathrm{T} 0338 \mathrm{~b}$ & & & $\mathrm{~T} 0447 \mathrm{c}$ & & T0493c \\
\hline T0231a & & & & T0258a & & & & Т0448a & & \\
\hline T0232a & & & & T0259a & & & & Т0449a & & \\
\hline T0233a & & & & T0260a & & & & T0449e & & \\
\hline
\end{tabular}


Family CO
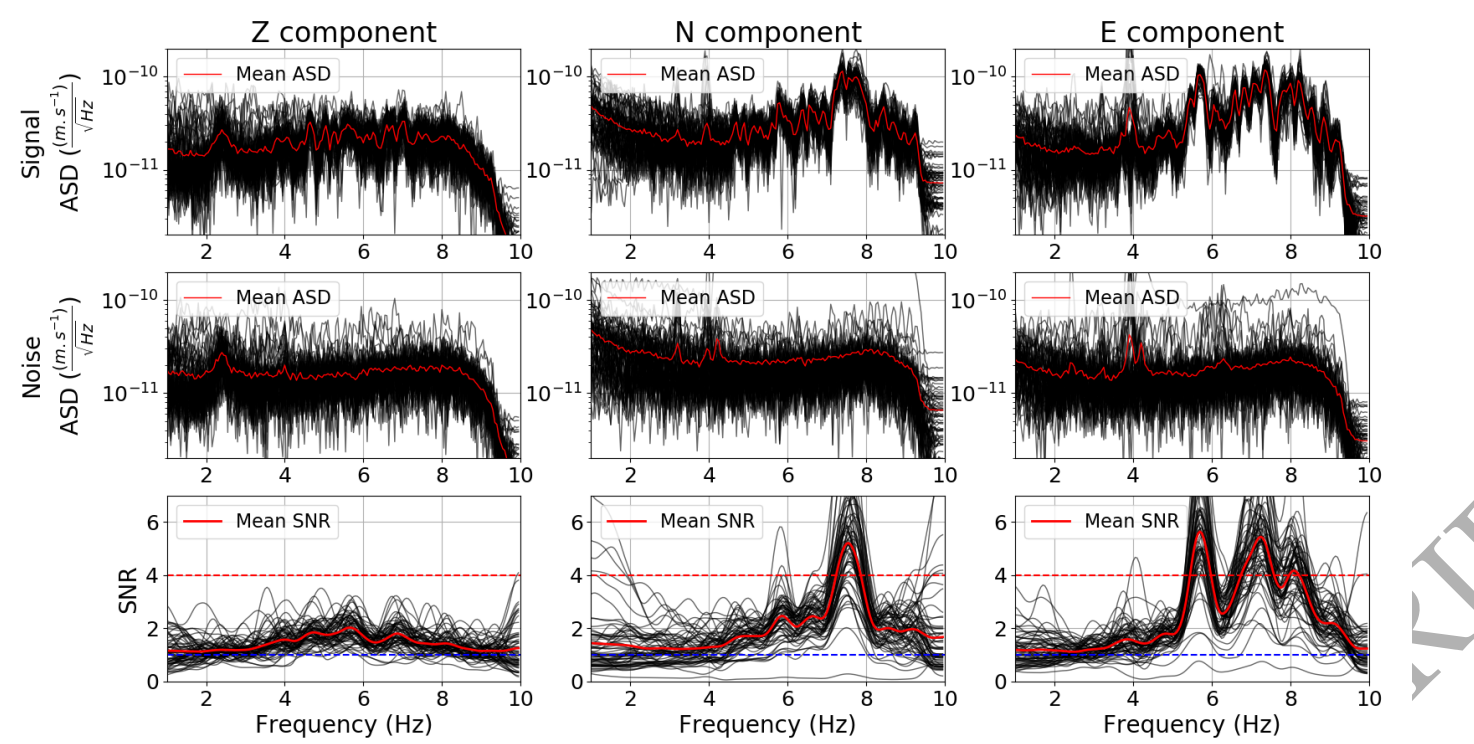

Family D0
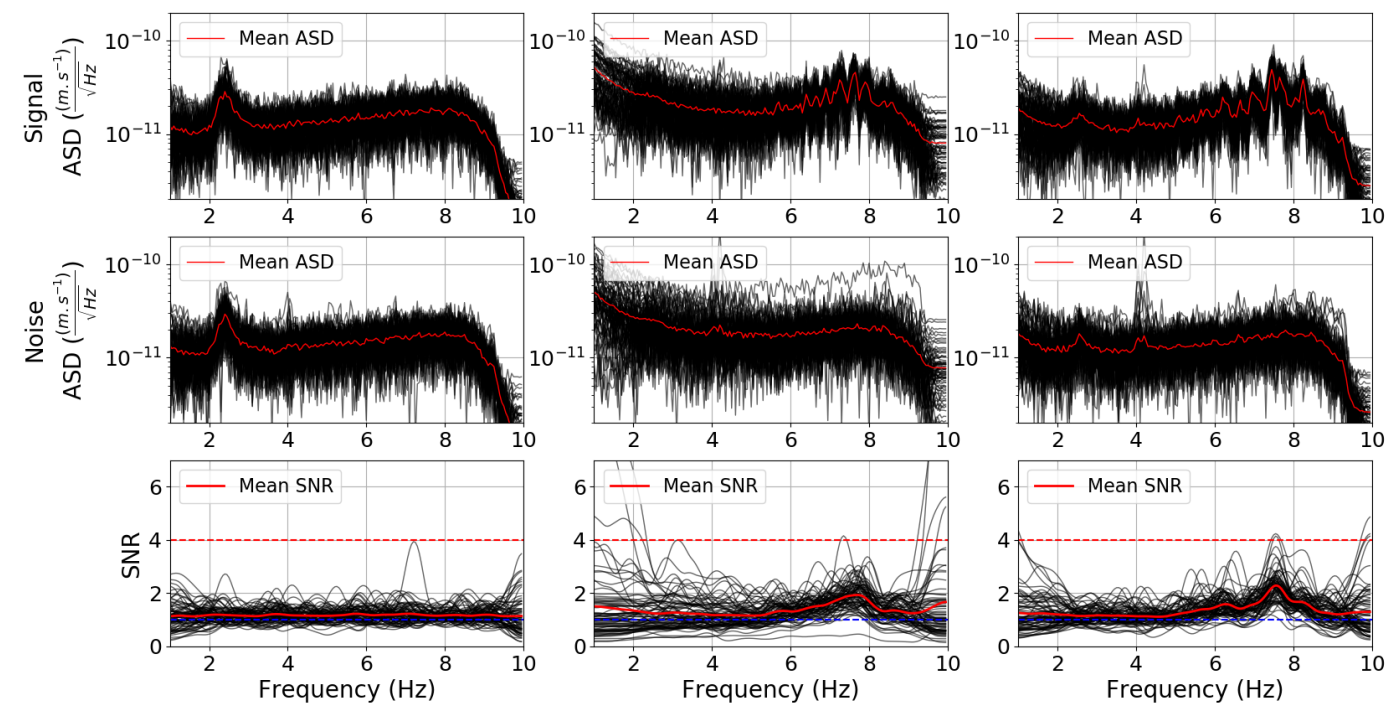

Figure A1. SRN analysis in the frequency domain performed on the C0 and D0 families. In each panel the ASD or SNR for all the event of the given family are shown in black. The mean ASD or mean SNR are shown in red. Two SNR thresholds are displayed in their corresponding panel in blue for $S N R=1$ and in red for $S N R=4$. 
Family C1
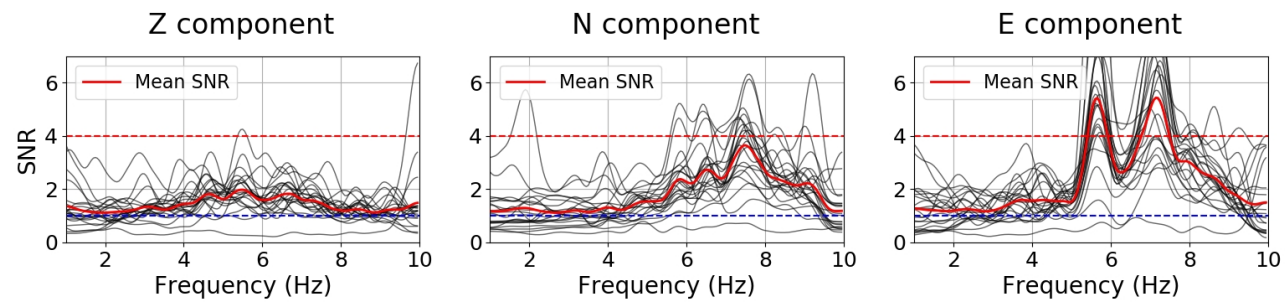

Family D1
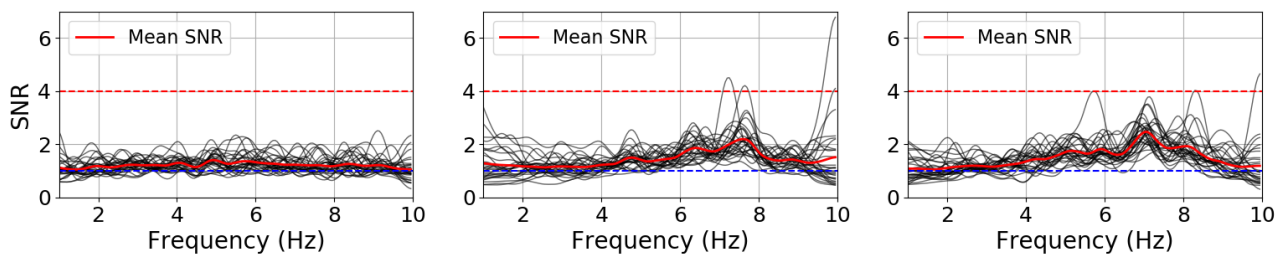

Family D2
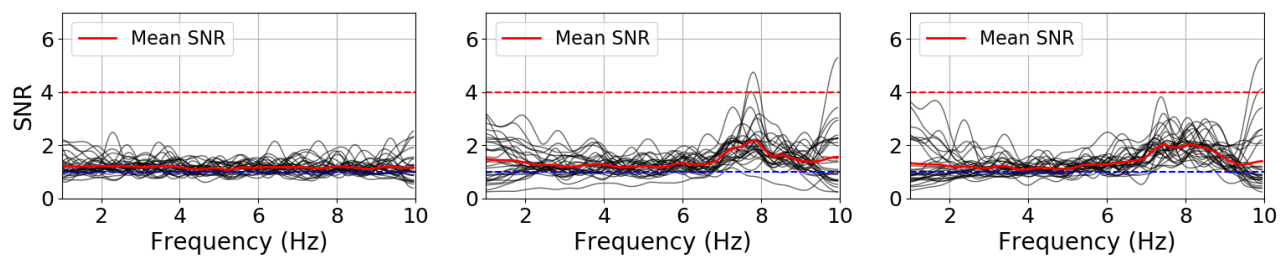

Family D3
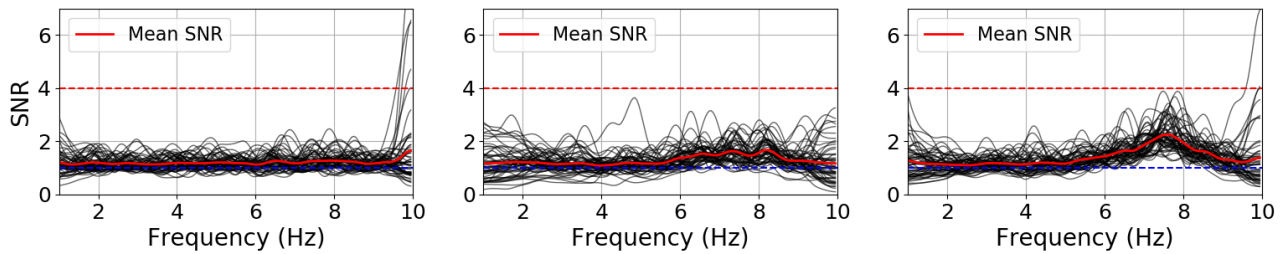

Family D4
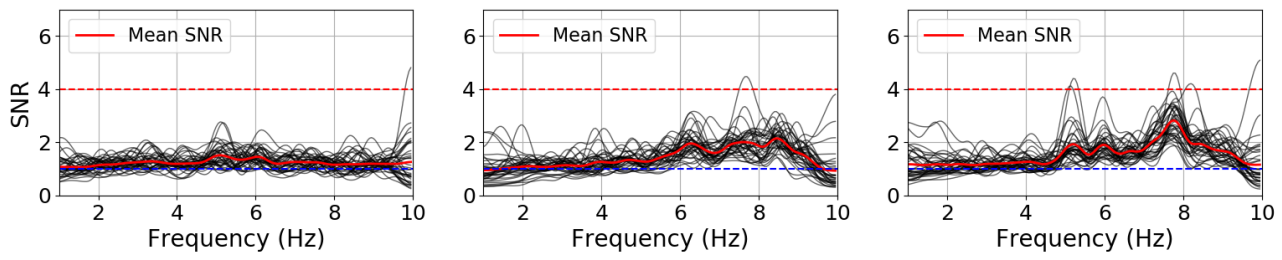

Family C2
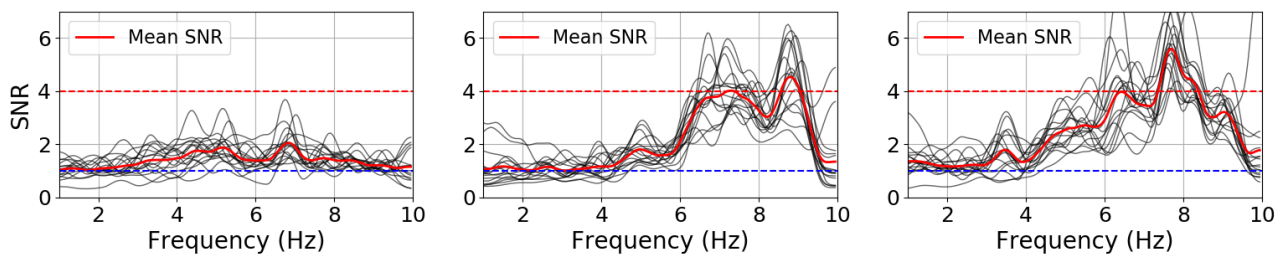

Figure A2. Same analysis than in Figure A1 for the C1, D1, D2, D3, D4 and C2 families. Here only the resulting SNR are shown. 

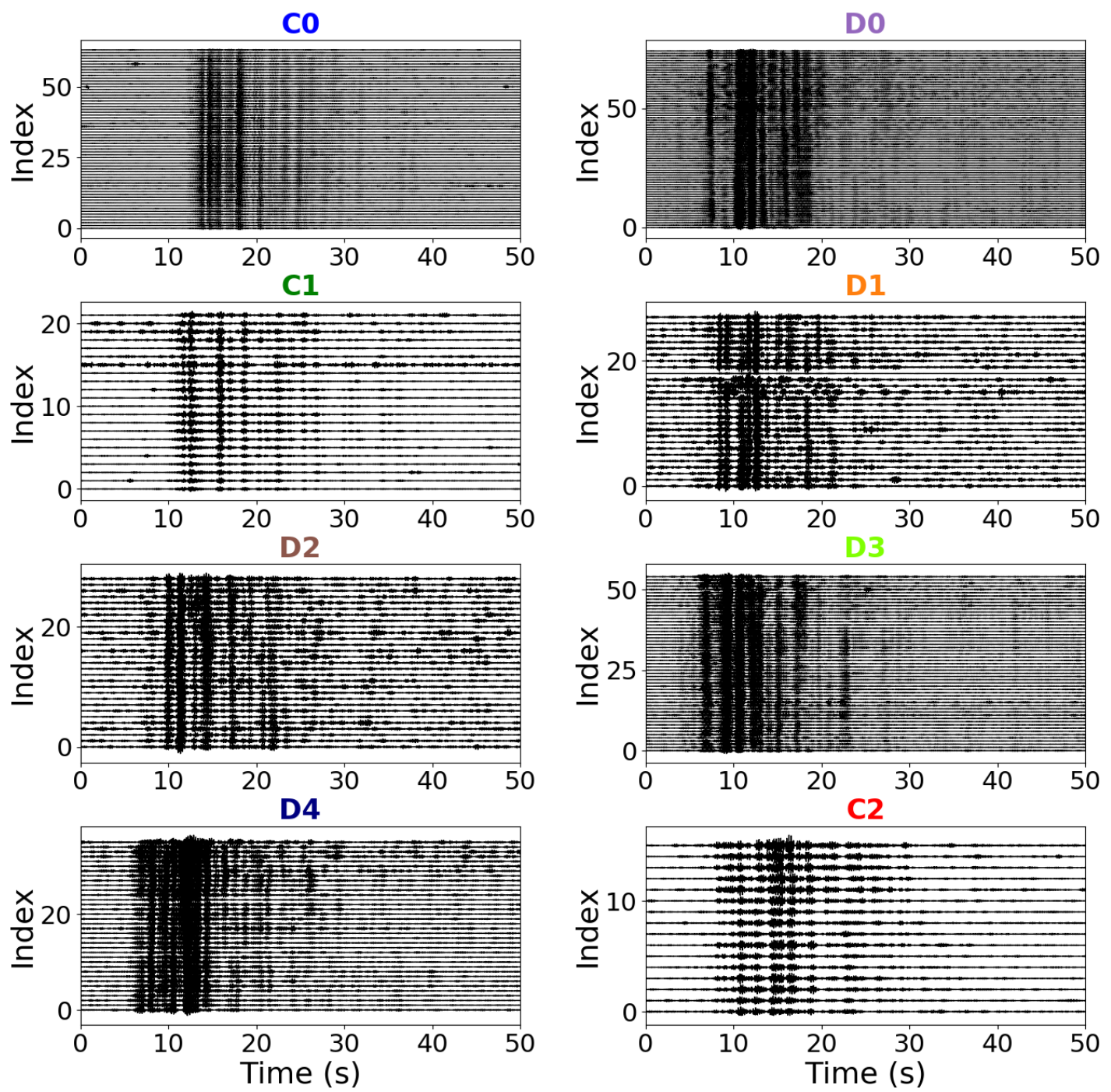

Figure A3. Waveforms of the East component filtered between $7 \mathrm{~Hz}$ and $9 \mathrm{~Hz}$ of the SF events in each family ordered by increasing Sols.

\section{A2 Waveforms}

We present in Figure A3 the waveforms of the SF events in each family indexed by increasing Sols.

\section{APPENDIX B: PSD OSCILLATION : EAST AND VERTICAL COMPONENT}

We show in Figure A4 the PSD oscillations of the background vibrations recorded on the VBB East and Vertical components. The Figure A4 and the Figure 5 in section 3.1 show that the band structure between $5 \mathrm{~Hz}$ and $10 \mathrm{~Hz}$ is only recovered on the horizontal components of SEIS. 


\section{East Component}

A)

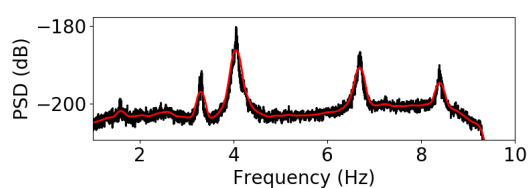

C)

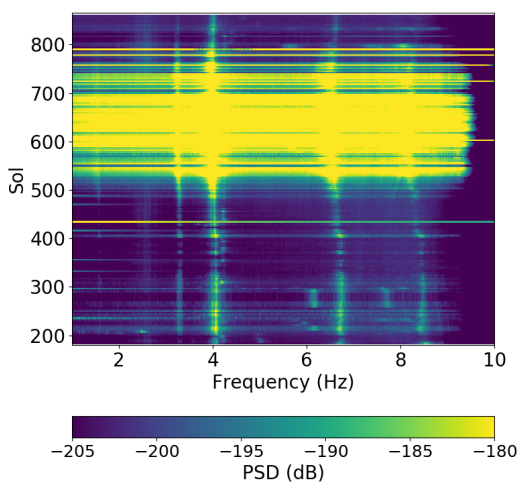

B)

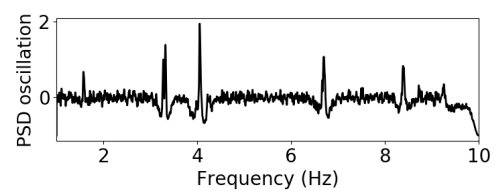

D)

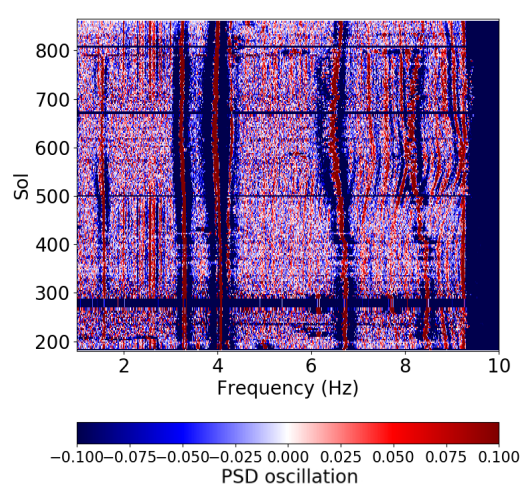

\section{Vertical Component}

E)

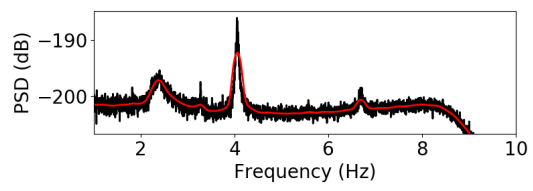

G)

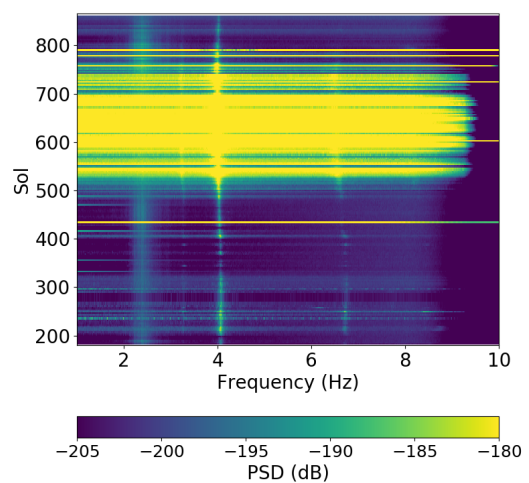

F)

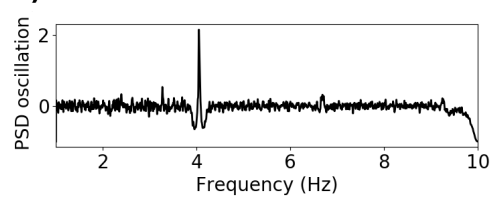

H)

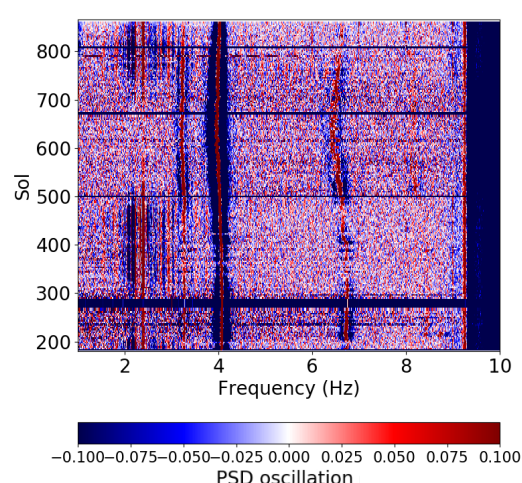

Figure A4. A) Power Spectral Density (PSD) in dB of the VBB East component between 18h and 23h LMST of Sol 350 (black curve) and its smoothed version (red curve) with a smoothing parameter of $0.32 \mathrm{~Hz}$. B) Same PSD as in panel A after whitening and centering (PSD fluctuation). C) Evening spectrogram of the VBB East component between the Sol 180 and Sol 866. D) Same spectrogram as in panel C after whitening and recentering. (E, F, G, H) Same analysis than previously for the VBB vertical component. 


\section{APPENDIX C: TEMPERATURE RECORDS AT INSIGHT}

The two temperatures records used in sections 4 and 5 are described hereafter :

- Surface temperature T2 from radiometer RAD (Kopp et al. 2016; Mueller et al. 2020) : The infrared radiometer RAD (part of the HP3-RAD experiment) mounted on the deck of the InSight lander measures the surface brightness in two areas close to the lander (at 1 and 3 meters). The surface temperature $\mathrm{T} 2$ is derived from the measurements in the area furthest from the lander. This area is supposed to be the less sensitive to the perturbation induced by the shadow of the lander (Siegler et al. 2017). The radiometer measurements are not continuous. The normal mode of operation is to acquire data at every full hour, although there is often a 2 hour gap in the evening due to operational reasons. Usually measurements are acquired for at least $24 \mathrm{~h}$, starting around noon, so that the observations stretch over at least 2 Sols. To generate the daily average temperature from these data we generate complete diurnal curves sampled at 144 equal intervals by combining data from two adjacent Sols and interpolating over data gaps of 2 hours or less. Only the average temperature of complete diurnal curves are presented here.

- SCIT (SCIentific Temperature) : Temperature of the air inside the Remote Warm Enclosure Box (RWEB) of the SEIS instrument, i.e. inside the first thermal barrier made of Titanium and Mylar and the second thermal barrier : the Wind and Thermal Shield (WTS) (Lognonn et al. 2019). Consequently the SCIT is shifted of approximately 7 hours with the atmospheric temperature (Pou et al. 2018). Nevertheless the seasonal variations of the daily average SCIT is very similar in both phase and amplitude to the temperature measurements derived from the radiometer RAD (see Fig. 9.A). We thus consider the SCIT as a good approximation of the surface temperature at period where the data from the RAD instrument are not available.

\section{APPENDIX D: THERMOELASTIC STRESS}

In 2D Cartesian geometry, the thermoelastic problem can be tackled by the introduction of the Airys stress function, $\psi$, that is related to the stress components as follows (Berger 1975): $\sigma_{x x}=\partial_{z z}^{2} \psi$, $\sigma_{z z}=\partial_{x x}^{2} \psi$ and $\sigma_{x z}=-\partial_{x z}^{2} \psi$. We recall that $x$ and $z$ refer to axes that are parallel and perpendicular to the surface, respectively. Airys function obeys a biharmonic equation with a source term related to the temperature field $T$ :

$$
\nabla^{4} \psi+\frac{E \alpha}{1-\nu} \nabla^{2} T=0
$$

where $\alpha$ is the thermal expansion coefficient, $E$ is Young's modulus and $\nu$ is Poisson's ratio. The harmonic solution obtained for zero normal and tangential stresses at the surface $\sigma_{z z}=\sigma_{x z}=0$, and 
with stresses vanishing at infinite depth is given by:

$$
\begin{gathered}
\psi(x, z, t)=\frac{\alpha E}{1-\nu} T_{\omega} \cos \left(k_{x} x\right)\left[\frac{k_{x}^{2} \cos \left(\omega t+\phi_{\omega}-\gamma z\right)-2 \gamma^{2} \sin \left(\omega t+\phi_{\omega}-\gamma z\right)}{4 \gamma^{4}+k_{x}^{4}} e^{-\gamma z}\right. \\
-\frac{k_{x}^{2} \cos \left(\omega t+\phi_{\omega}\right)-2 \gamma^{2} \sin \left(\omega t+\phi_{\omega}\right)}{4 \gamma^{4}+k_{x}^{4}} e^{-k_{x} z} \\
\left.-\frac{\left(\gamma+k_{x}\right) \cos \left(\omega t+\phi_{\omega}\right)+\gamma \sin \left(\omega t+\phi_{\omega}\right)}{2 \gamma^{2}+2 \gamma k_{x}+k_{x}^{2}} z e^{-k_{x} z}\right] .
\end{gathered}
$$

The principal stress $\sigma$ is then given by :

$$
\begin{aligned}
& \sigma(x, z, t)=\frac{2 \alpha E}{1-\nu} T_{\omega} \cos (\left.k_{x} x\right)\left[\cos \left(\omega t+\phi_{\omega}-\gamma z\right) e^{-\gamma z}\right. \\
&\left.-(1+\nu) \frac{\left(\gamma+k_{x}\right) \cos \left(\omega t+\phi_{\omega}\right)+\gamma \sin \left(\omega t+\phi_{\omega}\right)}{2 \gamma^{2}+2 \gamma k_{x}+k_{x}^{2}} k_{x} e^{-k_{x} z}\right],
\end{aligned}
$$

where $T_{\omega}$ and $\phi_{\omega}$ are the amplitude and phase of the surface temperature oscillation at pulsation $\omega$. Note that compared to Richter et al. (2014), we did not assume that the horizontal wavenumber $k_{x}$ is small compared to inverse penetration depth $\gamma$.

\section{APPENDIX E: CASE $L_{X}=30$ METERS}

In this section we investigate the prediction of the thermoelastic model when the wavelength $l_{x}$ approaches the order of magnitude of the Rayleigh wavelength. With an average shear wave speed in the subsurface of $100 \mathrm{~m} / \mathrm{s}$, the Rayleigh wavelength varies between 26 meters at $8 \mathrm{~Hz}$ and 37 meters at 6 $\mathrm{Hz}$.

The parameters of the model are fixed as follows: thermal expansion coefficients $\alpha=3 \times$ $10^{-5} \mathrm{~K}^{-1}, \partial \rho v_{p}^{2} / \partial \sigma=\partial \rho v_{s}^{2} / \partial \sigma=-700$, thermal conductivity $\kappa=3.9 \times 10^{-2} \mathrm{~W} / \mathrm{m} / \mathrm{K}$, density $\rho=1800 \mathrm{~kg} / \mathrm{m}^{3}$, specific heat capacity $C_{p}=600 \mathrm{~J} / \mathrm{kg} / \mathrm{K}$, Poisson's ratio $\nu=0.2$ and $l_{x}=30$ meters. The surface temperature forcing (Fig. A5.A) and the temperature field perturbation in depth (Fig. A5.B) are the same than in section 4.2.

In Figure A5.C we show the velocity perturbation until 10 meters depth induced by changes in the surface thermal forcing with time (Sol) when $l_{x}=30 \mathrm{~m}$. We also show the sensitivity kernels for $\mathrm{P}$ - and $\mathrm{S}$-waves at 6 and $8 \mathrm{~Hz}$. We see that high amplitude perturbations propagate deeper than in the case $l_{x}=1000 \mathrm{~m}$. This have a direct impact on the $d v / v$ variations with $\mathrm{S}$-waves sensitivity. We can see in Figure A5.D that the S- $d v / v$ variation has, in that case, a negative correlation with the surface temperature,

Our observations of relative velocity changes and the Rayleigh wave assumption constrain the horizontal wavelenght $l_{x}$ to be, at least, one order of magnitude higher than the Rayleigh wavelenght. 


\section{过}
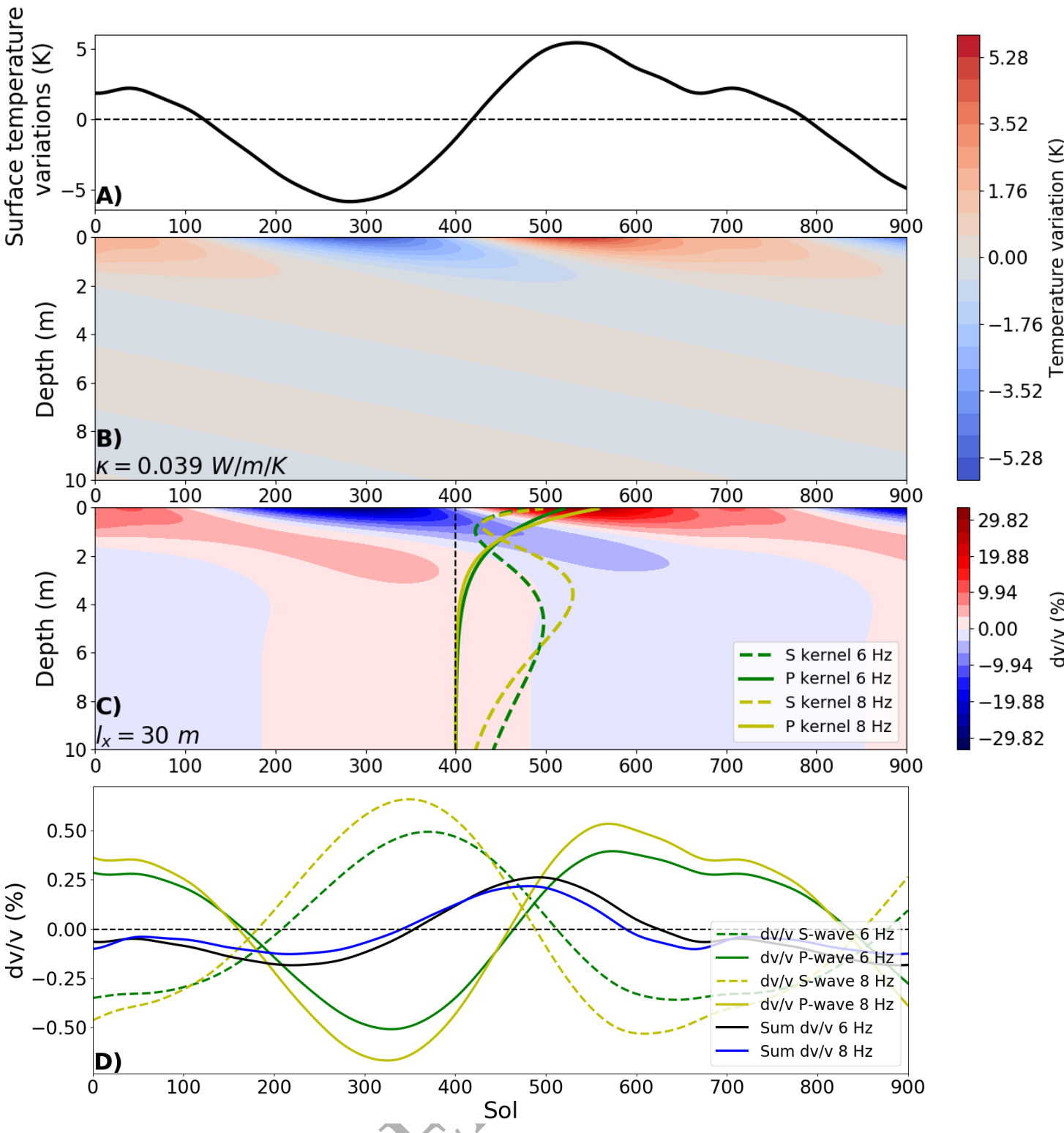

Figure A5. A)Surface temperature model (in Kelvin) calculated from the incident solar flux deduced from the JPL Horizon Ephemeris. B) Variation of the temperature field at depth as a function of time (expressed in Sol) caused by the variation of the incident solar flux considering a thermal conductivity $\kappa=0.039 \mathrm{~W} / \mathrm{m} / \mathrm{K}$. C) Relative velocity variation (\%) at depth induced by the temperature field perturbation shown in (B) as predicted by the thermoelastic model (Eq. (3)) with the same parameters as in Figure 8.C except for $l_{x}$, equal here to 30 meters. Superimposed on the velocity variation, we show the sensitivity kernels for P-waves (solid lines) and S-waves (dashed lines) at $6 \mathrm{~Hz}$ (green) and $8 \mathrm{~Hz}$ (yellow). D) Relative velocity variation predicted from P (solid lines) and $\mathrm{S}$ (dot lines) sensitivity at $6 \mathrm{~Hz}$ (green) and $8 \mathrm{~Hz}$ (yellow). The resulting velocity perturbations at 6 and $8 \mathrm{~Hz}$ are drawn in solid black and blue lines respectively. 